Full Length Article

\title{
The effect of quasi-free graphene layer on the electrical transport properties of sandwich-like graphene / Co nanoparticles / graphene structure
}

\author{
V. Bayev a, ${ }^{\text {, }}$ M. Rybin ${ }^{\mathrm{b}}$, I. Svito ${ }^{\mathrm{c}}$, J. Przewoźnik $^{\mathrm{d}}$, Cz. Kapusta ${ }^{\mathrm{d}}$, J. Kasiuk $^{\mathrm{a}, \mathrm{c}}$, S. Vorobyova ${ }^{\mathrm{e}}$, \\ A. Konakov e, f, E. Obraztsova ${ }^{b}$ \\ ${ }^{a}$ Institute for Nuclear Problems, Belarusian State University, Bobruiskaya str. 11, Minsk, Belarus \\ ${ }^{\mathrm{b}}$ A.M. Prokhorov General Physics Institute, Vavilov Str. 38, Moscow, Russia \\ ${ }^{\mathrm{c}}$ Energy Physics Department, Faculty of Physics of the Belarusian State University, Bobruyskaya str. 5, Minsk, Belarus \\ ${ }^{\mathrm{d}}$ Department of Solid State Physics, Faculty of Physics and Applied Computer Science, AGH University of Science and Technology, Krakow, Poland \\ ${ }^{e}$ Research Institute for Physical Chemical Problems of the Belarusian State University, Leningradskaya str. 14, Minsk, Belarus \\ ${ }^{\mathrm{f}}$ Institute of Problems of Chemical Physics of Russian Academy of Sciences (IPCP RAS), Academician Semenov avenue 1, Chernogolovka, Russian Federation
}

\section{A R T I C L E I N F O}

\section{Keywords:}

CVD graphene

Hall effect

Charge transport

Carrier mobility

Magnetotransport

Transparent conductive films

\begin{abstract}
A B S T R A C T
The paper reports on the charge transport in the sandwich-like graphene / Co nanoparticles / graphene structure (G/CoNPs/G). It is fabricated by combining the electrochemical deposition of Co nanoparticles onto a single layer CVD graphene and their capping with another graphene layer. The isolated semi-spherical, surface oxidized Co NPs of $250 \mathrm{~nm}$ in diameter serve as a support for the top graphene layer and provide its suspended state confirmed by atomic force microscopy revealing periodic ripples on its surface. A high optical transparency of $92.4 \%$ is found for the G/CoNPs/G sandwich. The observed twofold reduction in the sheet resistance with $\mathrm{a} \sim 1.5$ times increase in the positive magnetoresistive effect for G/CoNPs/G compared to the pristine graphene is attributed to the suppression of carrier scattering due to the substrate. From the Hall effect measurements, a predominant role of the top graphene layer in the charge transport in G/CoNPs/G is established, with a significantly increased carrier mobility $\mu \sim 1800 \mathrm{~cm}^{2} /(\mathrm{V} \cdot \mathrm{s})$. An elastic intervalley scattering length estimated from the magnetoresistance results is found to be three times increased in G/CoNPs/G, indicating that the top graphene layer is almost unaffected by the substrate. The proposed technology looks promising for possible optoelectronic and magneto-optical applications.
\end{abstract}

\section{Introduction}

Transparent conductive films (TCFs) play an exceptional role in many modern applications that use liquid crystal displays, organic light-emitting diodes (OLED) or photovoltaic cells. In addition, TCFs are widely used in fabrication of electromagnetic interference shielding, antistatic and antiglare coatings. Possessing an optimal set of properties (high optical transparency exceeding $90 \%$ and electrical conductivity of $10-30 \mathrm{Ohm} / \mathrm{square}$ ), Indium Tin Oxide (ITO) has become the market standard for most of these applications [1].

The use of ITO may be limited for both technical and economic reasons. Indium is a relatively expensive rare metal, with ITO synthesis methods (sputtering, evaporation, pulsed laser deposition and electroplating) being rather energy-consuming. Considering the trends in indium demand, levels of its production and recycling, in 20-30 years the existing supply may hardly meet the demand. At this point, the price will rise [2]. Moreover, ITO is a delicate crystalline material that excludes its use as a flexible TCF. At the same time, fabrication of flexible TCFs is the primary task for producing flexible OLED displays. The most promising materials in this field are carbon nanotubes [3,4], metal nanowires [5], conducting polymers [6,7], and graphene [1].

There are several factors to consider when choosing an appropriate material for application as flexible TCFs, which include the surface roughness of the films, the optimal ratio between electrical conductivity and optical transparency, as well as manufacturing costs. In this regard, graphene seems to be a good candidate. A commonly-used and cost-effective method for the graphene synthesis is chemical vapor deposition (CVD) with copper foil used as a substrate. The graphene can be put onto an insulating substrate (silicon oxide or glass) to measure its transport properties. CVD graphene has a polycrystalline structure consisting of crystallites with a size of about $1 \mu \mathrm{m}$ and grain boundaries, which unfortunately reduce significantly its electrical conductiv-

\footnotetext{
* Corresponding author.

E-mail address: vadim.gh.bayev@gmail.com (V. Bayev).
} 
ity. The average sheet resistance of a single layer CVD graphene is about $1000 \mathrm{Ohm} / \mathrm{square}$, which hinders its use for application in flexible TCFs.

In 2015, a research group from the Republic of Korea (Electronics and Telecommunications Research Institute) designed a process that can pattern a graphene-made transparent electrode on a glass substrate [8]. Later in 2017, a flexible OLED panel produced using graphene on a plastic flexible substrate was demonstrated. The researchers developed a "fully operational" OLED panel that uses a pixelated graphene film as transparent electrodes [9]. At the moment, this group continues to improve this technology for commercial use [10].

According to the data presented in [11], high graphene resistance leads to an increase in the required applied voltage at the OLED pixel that appears in the problem of achieving luminance uniformity over OLED lighting panels of a large area. However, the authors have successfully realized a prototype of the OLED display using multilayered graphene with a sheet resistance of $250 \mathrm{Ohm} /$ square. The further development of this technology is closely related to a conductivity increase of the graphene used, preserving its transparency close to 85-90\%. For commercial application of graphene as an electrode in the OLED technology, its sheet resistance is needed to be reduced down to $50 \mathrm{Ohm} /$ square. There are several known methods for decreasing the electrical resistance of the CVD graphene: doping, combination with metal nanowires and fabrication of complex multilayered graphene-based structures [12].

The doping is the most studied method of electrical properties modification of CVD graphene. For example, the effect of doping of graphene with bis(trifluoromethanesulfonyl)amide was investigated in [13] for the structures with different number of graphene layers. The authors managed to reduce the sheet resistance of such structures to $240 \mathrm{Ohm} /$ square for single layer graphene and to $90 \mathrm{Ohm} /$ square for the system containing five graphene layers, with a resulting transparency of $88 \%$. The methods of doping of CVD graphene with $\mathrm{AuCl}_{3}$ in nitromethane [14], $\mathrm{FeCl}_{3}$ [15] and $\mathrm{CuCl}$ [16] were proposed. These dopings resulted in a sheet resistance of about $150 \mathrm{Ohm} /$ square and with a transparency of $87 \%$. However, it should be noted that the results obtained do not take into account the stability of the attained parameters in the long term [15].

An alternative method of improving the electrical conductivity of the CVD-graphene is the combination of graphene layers with metal nanowires. For example, the CVD graphene modification with silver nanowires was proposed in [17]. The sheet resistance for the initial single layer CVD graphene was found to be $\sim 800 \mathrm{Ohm}$ /square. The combination of the graphene layers with silver nanowires reduced the values of the sheet resistance down to $24 \mathrm{Ohm}$ /square, with the transparency of the obtained composite reaching $90 \%$. A decrease in the sheet resistance is achieved by shunting the grain boundaries with metal nanowires. Thus, it is shown that the influence of intergranular space in CVD graphene on its sheet resistance can be reduced by nanoscale metal inclusions.

The electrochemical deposition of Co nanoparticles (NPs) onto the surface of a twisted multilayered CVD graphene was described previously in [18] as a method of modification. A complex analysis revealed the formation of isolated quasi-spherical partly-oxidized Co NPs with an average diameter of $220 \mathrm{~nm}$ and a core-shell structure as a result of such a deposition. It was shown that the core of the deposited NPs contains Co metal while the shell comprises an oxide CoO. Recently, a significant decrease in the sheet resistance was reported in the twisted multilayered CVD graphene modified with electrochemically deposited Co nanoparticles (from $2300 \mathrm{Ohm} / \mathrm{square}$ to $300 \mathrm{Ohm} / \mathrm{square}$ ) [19]. A decrease in sheet resistance is associated with the shunting of grain boundaries by metal nanoparticles.

The transparency of graphene before the electrochemical deposition of cobalt was found to be $97 \%[16,20]$, with the subsequently deposited Co NPs occupying less than $5 \%$ of the sample area according to the pro- cessing of the images of scanning electron microscopy $[18,19]$. Therefore, the resulting optical transparency of the composite structures is expected to be about $92 \%$ that indicates a great potential of electrochemically modified graphene in designing flexible TCFs.

In the present paper, we describe the modification of single layer CVD graphene, with electrochemically deposited Co NPs on its surface, by covering it on top with an additional single layer of CVD graphene (sandwich-like structure "graphene/Co NPs/graphene" will be marked briefly as G/CoNPs/G hereafter). As it was reported in [21,22], additional deposition of CVD graphene monolayer allows to obtain a socalled turbostratic stacked graphene with enhanced charge carrier mobility and conductivity similar to those of suspended graphene, which is a consequence of reduction of carrier scattering in the top graphene layer. In our case, we suppose that Co NPs with $\mathrm{CoO}$ shells deposited onto the bottom graphene layer could be successfully used as a point support for the top layer in order to eliminate the substrate effect on the conductivity (such as scattering by charged impurities and surface phonons of substrate), thus improving the electrical transport properties of the top graphene layer. The expected high optical transparency of this sandwich-like structure of $\sim 90 \%$ allows to consider it as a promising composite for the development of flexible TCFs for optoelectronic and magneto-optical applications, accounting for the magnetic nature of Co NPs.

\section{Experimental}

\subsection{Materials}

Sheets of single layer CVD graphene with a size of $10 \times 10 \mathrm{~mm}$ were fabricated by cold-wall chemical vapor deposition on the surface of $25 \mu \mathrm{m}$ thick copper foil ( $99.8 \%$ purity) from the methane precursor with nitrogen as a carrier gas. The procedure of graphene fabrication and its characterization is described in details in [23]. The sheets of graphene were transferred onto $\mathrm{SiO}_{2} / \mathrm{Si}$ substrate using standard procedure of poly(methyl methacrylate) (PMMA) spin-coating and further dissolving of $\mathrm{Cu}$ substrate by ammonium persulfate solution. The PMMA/graphene membrane is scooped onto the $\mathrm{SiO}_{2} / \mathrm{Si}$ substrate. The PMMA layer was removed with acetone.

Electrochemical deposition of cobalt onto the surface of graphene on the $\mathrm{SiO}_{2} / \mathrm{Si}$ substrate was carried out at a constant current at room temperature, similarly as it was described in [18]. The $\mathrm{SiO}_{2} / \mathrm{Si}$ substrate with a size of $10 \mathrm{~mm} \times 4 \mathrm{~mm}$, covered with CVD graphene layer, was used as a cathode that is electrically connected with copper wire by indium solder, while the platinum plate was used as an anode, with the distance between the electrodes being about $5 \mathrm{~mm}$. The $\mathrm{Ag} / \mathrm{AgCl}$ electrode was used as a reference. The electrolyte contained $3.5 \mathrm{mM}$ of cobalt (II) sulphate heptahydrate $\left(\mathrm{CoSO}_{4} \cdot 7 \mathrm{H}_{2} \mathrm{O}\right)$ and $1 \mathrm{mM}$ of sodium chloride $(\mathrm{NaCl})$ dissolved in distilled water. All the reagents used for electrochemical deposition were of analytical grade and no additional purification was applied.

The cyclic voltammetry process was performed in the classical three-electrode configuration with $\mathrm{Ag} / \mathrm{AgCl}$ reference electrode to determine the optimal current density for Co NPs deposition. As shown in Fig. 1, the reduction of $\mathrm{Co}^{2+}$ at the CVD graphene on $\mathrm{SiO}_{2} / \mathrm{Si}$ substrate is observed in the range of potential $E$ from $-0.9 \mathrm{~V}$ to $-1.5 \mathrm{~V}$ determining the formation of Co NPs. Further decrease in $E$ value below $-1.6 \mathrm{~V}$ leads to a hydrogen evolution reaction. The dissolution of cobalt is observed in the potential range from -0.6 to $-0.1 \mathrm{~V}$. The obtained results of cyclic voltammetry are in a good agreement with published data on the electrochemical reduction of cobalt on glassy carbon using the electrolyte of similar composition [24]. Thus, the current density of $2.5 \mathrm{~mA} / \mathrm{cm}^{2}$ corresponding to the potential region from $-0.8 \mathrm{~V}$ to $-1.6 \mathrm{~V}$ was chosen as the most optimal for the predominant reduction of $\mathrm{Co}^{2+}$ at the CVD graphene on $\mathrm{SiO}_{2} / \mathrm{Si}$ substrate used as a cathode. After the process of electrochemical deposition of Co NPs for $30 \mathrm{~s}$, 


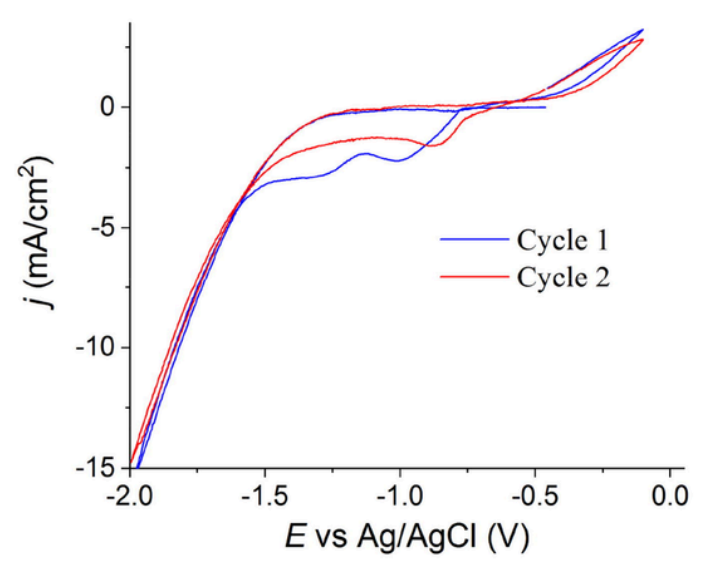

Fig. 1. Cyclic voltammetry of CVD graphene/SiO/Si with scan rate $2 \mathrm{mV} / \mathrm{s}$ in electrolyte containing $3.5 \mathrm{mM} \mathrm{CoSO} \mathrm{C}_{4} \cdot 7 \mathrm{H}_{2} \mathrm{O}$ and $1 \mathrm{mM} \mathrm{NaCl}$ dissolved in distilled water.

the obtained samples of graphene modified with Co NPs (G/CoNPs) were thoroughly washed with distilled water and dried at room temperature.

The sandwich-like structure G/CoNPs/G was obtained by transfer of a pristine single layer CVD graphene onto G/CoNPs composite on $\mathrm{SiO}_{2} / \mathrm{Si}$ substrate using a complex procedure including standard PMMA spin-coating of graphene on the $\mathrm{Cu}$ foil, further dissolving of $\mathrm{Cu}$ substrate by ammonium persulfate solution, a PMMA/graphene membrane transfer onto the G/CoNPs composite on $\mathrm{SiO}_{2} / \mathrm{Si}$ substrate, and the PMMA layer removal with acetone. The full process is illustrated at the sketch in Fig. 2. In order to measure the light transmission spectra of a single layer CVD graphene, G/CoNPs and G/CoNPs/G samples, the pro- cedure of sample preparation described above was repeated using a glass substrate instead of the $\mathrm{SiO}_{2} / \mathrm{Si}$ substrate.

\subsection{Methods}

Raman spectra were obtained using a Horiba LabRAM HR evolution Raman spectrometer equipped with $30 \mathrm{~mW}$ laser operating at a wavelength of $532 \mathrm{~nm}$. The results of Raman spectroscopy were verified by measuring three different regions of each sample. Optical transparency was analyzed with a Perkin Elmer Lambda 950 optical absorption spectrometer. The microstructure was examined by scanning electron microscopy (SEM) using a $L E O-1455 \mathrm{VP}$ microscope operating at a voltage of $20 \mathrm{kV}$. It is equipped with the nitrogen-free Aztec Energy Advanced XMax 80 energy dispersive X-ray (EDX) spectrometer, which was used for obtaining the maps of elemental composition of the materials. Surface morphology was analyzed with an NT-MDT Solver-Pro 47 atomic force microscope (AFM) using a super sharp diamond-like NSG01 DLC carbon tip with the curvature radius less than $3 \mathrm{~nm}$.

Electrical transport properties (the four-probe resistance $(R)$, magnetoresistance $(M R)$, and four-probe Hall effect) were measured using the resistivity options of a Quantum Design Physical Property Measurement System (PPMS) and Cryogenic Ltd Cryogen-Free Measurement System (CFMS) at temperatures $2-300 \mathrm{~K}$ with the magnetic field up to $9 \mathrm{~T}$ applied along the normal to the sample plane. The samples were mounted on the removable contact pad of the probe using 6 indium (In) electrical contacts with soldered thin copper rods. The temperature was stabilized with the precision of $0.01 \mathrm{~K}$ using LakeShore 331 controller. The error in measuring the resistance was mainly due to the large size of electrical contacts and inter-contact distances and was estimated at 5 $\%$.

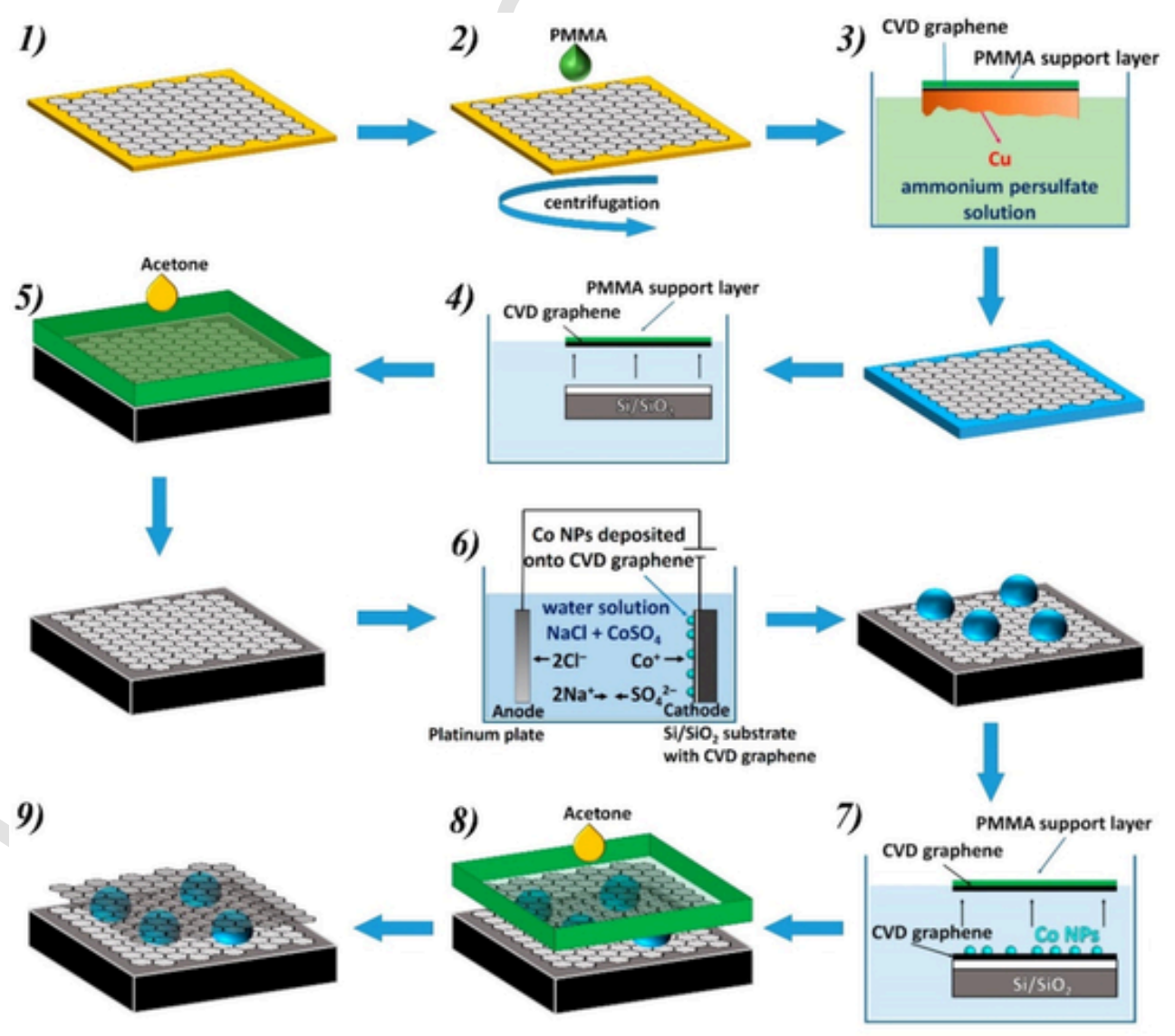

Fig. 2. The sketch of the process of synthesis of the sandwich-like G/CoNPs/G structure. 


\section{Results and discussion}

\subsection{Structure and surface morphology}

Raman spectra of the pristine graphene, G/CoNPs and G/CoNPs/G samples on the $\mathrm{SiO}_{2} / \mathrm{Si}$ substrate are presented in Fig. 3a. The main parameters describing the D-, G- and 2D-peaks of graphene seen in Fig. 3a are given in Table 1. The Raman spectra of the samples on the glass substrate were found to be identical to those on the $\mathrm{SiO}_{2} / \mathrm{Si}$ substrate. In addition, they do not reveal any significant changes in the pristine graphene structure after electrochemical deposition of Co NPs (sample $\mathrm{G} / \mathrm{CoNPs}$ ) and their further covering with the second graphene layer (sample G/CoNPs/G). The observed small shifts of G-peak $\left(\Delta_{\mathrm{G}}=1.3 \mathrm{~cm}^{-1}\right)$ and 2D-peak $\left(\Delta_{2 \mathrm{D}}=2.0 \mathrm{~cm}^{-1}\right)$ could be attributed to some strains, contaminations or doping effect $[16,25]$.

Optical transmission spectra of the graphene, G/CoNPs and G/ CoNPs/G samples on the glass substrate are represented in Fig. 3b. The graphene sample on the glass substrate shows the optical transmittance of $96.4 \%$ at normal incidence for light with a wavelength of $550 \mathrm{~nm}$. This value is very close to that for a single layer graphene, $97.7 \%$, cited in literature [26-28] or $97 \%$ reported for the single layer graphene synthesized with the same technique [16]. A possible reason of a slightly lower optical transmittance observed could be the presence of PMMA residues, which were not removed with acetone at the stage of graphene transfer. The G/CoNPs sample demonstrates distinctly lower optical transmittance of $94.2 \%$. The decrease in transmittance is most likely related to the presence of the optically opaque Co NPs at the surface of graphene. For the G/CoNPs/G sample, the optical transmittance (at $550 \mathrm{~nm}$ wavelength) is further reduced to $92.4 \%$. This reduction is associated with the deposition of the second layer of graphene.

SEM image of the sample surface after electrochemical deposition of Co NPs (G/CoNPs sample, shown in Fig. 4a) indicates that graphene favors the formation of isolated quasi-spherical NPs with a diameter of $250 \mathrm{~nm}$. The Co distribution determined along the line of EDX scan proves that nanoparticles observed by SEM contain Co. As it was shown earlier, the Co NPs deposited at similar conditions consist of metal Co cores covered by the CoO shells [18].

The results of SEM and EDX scanning of the G/CoNPs sample after its covering with the top graphene layer (G/CoNPs/G sample) are presented in Fig. 4b. The NPs observed in Fig. 4a remain at the surface of the bottom graphene layer but are registered with a clearly lower SEM contrast due to the absorption of electrons by the top graphene layer. The overall Co content in the deposited NPs is confirmed by EDX analysis.

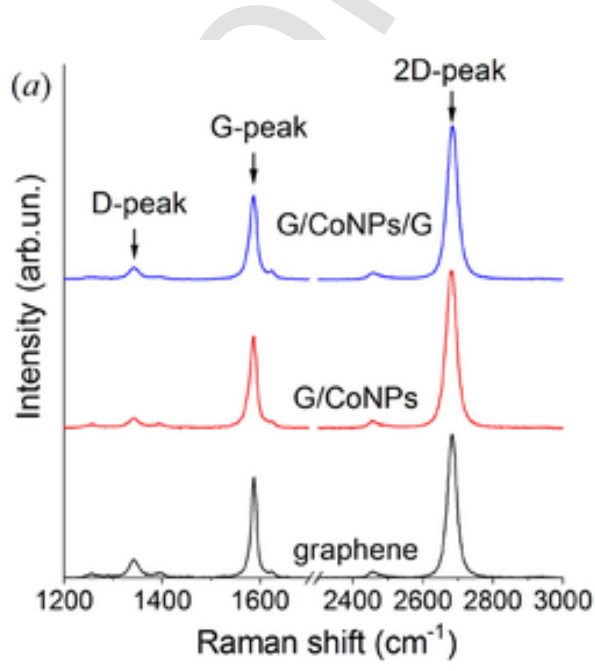

According to the AFM images of the G/CoNPs sample shown in Fig. 5 the diameter of Co nanoparticles is about $250 \mathrm{~nm}$, which correlates with the SEM results, while the average height of the particles appears to be of $100 \mathrm{~nm}$. It allows us to infer that the deposited Co NPs posess hemispherical shapes as shown in to Fig. 5 (right).

After covering the G/CoNPs sample with the top graphene layer (G/ CoNPs/G sample), AFM images reveal a significant decrease in the average height of the covered particles down to $30 \mathrm{~nm}$ (see Fig. 6). We suppose that this effect is most likely due to the suspended state of the top graphene layer (in G/CoNPs/G structure) on Co NPs at some height above the bottom graphene layer, while the Co NPs play a role of spacers. Indeed, an aspect ratio (height/lateral dimension) for the Co NPs in G/CoNPs srtucture is found to be about 0.4 that exceeds an estimated limit (0.1) for transition of covering them graphene layer from the supported to a partially suspended state [29]. Also it should be noted that the surface of the top graphene layer reveals periodic ripples with a height of $\sim 1 \mathrm{~nm}$, which are not observed for the G/CoNPs sample. Such ripples were earlier detected in suspended graphene membranes $[30,31]$.

\subsection{Electrical transport properties}

The four-probe electrical resistance as a function of temperature of the pristine and modified graphene on the $\mathrm{SiO}_{2} / \mathrm{Si}$ substrate was measured at temperatures of $2-300 \mathrm{~K}$ in a magnetic field $B$ perpendicular to the surface of samples. A constant electric current of $10 \mu \mathrm{A}$ was applied using current-carrying contacts $(I)$, and the value of potential difference was measured using voltage-sensing contacts $(U)$, as it is shown in the scheme of electrical contacts arrangement in Fig. 7a. The values of electrical resistance are presented in sheet resistance units (Ohm/ square) calculated as $R_{\mathrm{sq}}=R \cdot(W / L)$, where $R=U / I, W(\sim 4 \mathrm{~mm})$ is the distance between voltage-sensing contacts $U_{\mathrm{H}}, L(\sim 10 \mathrm{~mm})$ - distance between voltage-sensing contacts $U$.

The $R_{\mathrm{sq}}(T)$ dependences for the pristine and modified graphene on the $\mathrm{SiO}_{2} / \mathrm{Si}$ substrates are presented in Fig. 7b. At low temperatures (up to $\sim 170 \mathrm{~K}$ ), the resistance of graphene decreases with increasing temperature, evidencing the activation (semiconducting) type of electrical conductivity in the samples studied. Such a behavior can be assigned to the polycrystalline structure where crystallites of graphene with low resistance are separated by high-defect grain boundaries [32]. At temperatures higher than $170 \mathrm{~K}$, the character of $R_{\mathrm{sq}}(T)$ dependences changes. A slow growth of $R_{\mathrm{sq}}$ with increasing temperature is observed, indicating the dominance of quasi-metallic type of conductivity generally in-

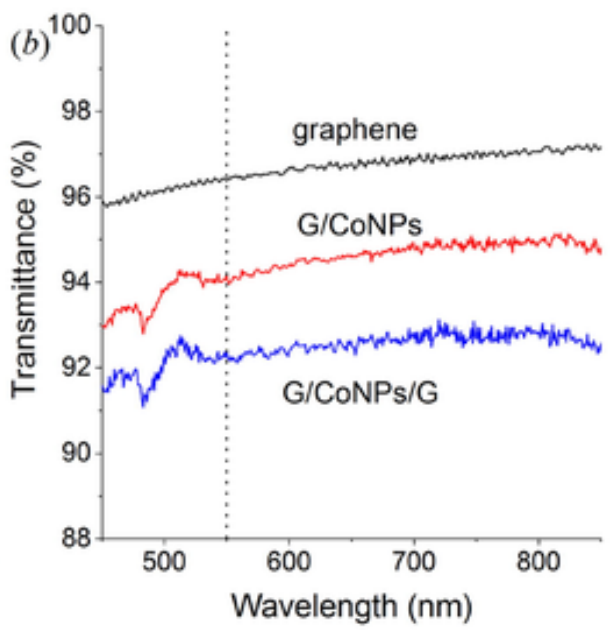

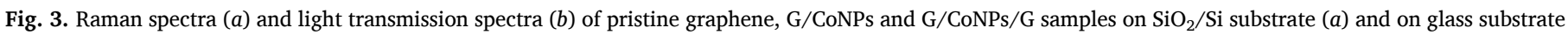
(b). 
Table 1

The parameters of D-, G- and 2D-peaks of graphene derived from Raman spectra: the integrated intensities of peaks in each spectrum are calculated with respect to the intensity of the corresponding 2D-peak.

\begin{tabular}{|c|c|c|c|c|}
\hline \multicolumn{2}{|c|}{ Parameters of the spectral lines } & \multirow{2}{*}{$\begin{array}{c}\text { graphene } \\
1342.6\end{array}$} & \multirow{2}{*}{$\begin{array}{l}\text { G/CoNPs } \\
1342.4\end{array}$} & \multirow{2}{*}{$\begin{array}{l}\text { G/CoNPs/G } \\
1342.8\end{array}$} \\
\hline D-peak & Raman shift, $\mathrm{cm}^{-1}$ & & & \\
\hline & FWHM, $\mathrm{cm}^{-1}$ & 22.0 & 24.6 & 24.1 \\
\hline & Intensity, \% & 9 & 4 & 6 \\
\hline \multirow[t]{3}{*}{ G-peak } & Raman shift, $\mathrm{cm}^{-1}$ & 1587.2 & 1585.9 & 1586.0 \\
\hline & FWHM, $\mathrm{cm}^{-1}$ & 12.6 & 16.8 & 17.4 \\
\hline & Intensity, \% & 31 & 29 & 30 \\
\hline \multirow[t]{3}{*}{ 2D-peak } & Raman shift, $\mathrm{cm}^{-1}$ & 2683.2 & 2681.2 & 2684.2 \\
\hline & FWHM, $\mathrm{cm}^{-1}$ & 27.8 & 31.7 & 30.7 \\
\hline & Intensity, \% & 100 & 100 & 100 \\
\hline
\end{tabular}

(a)
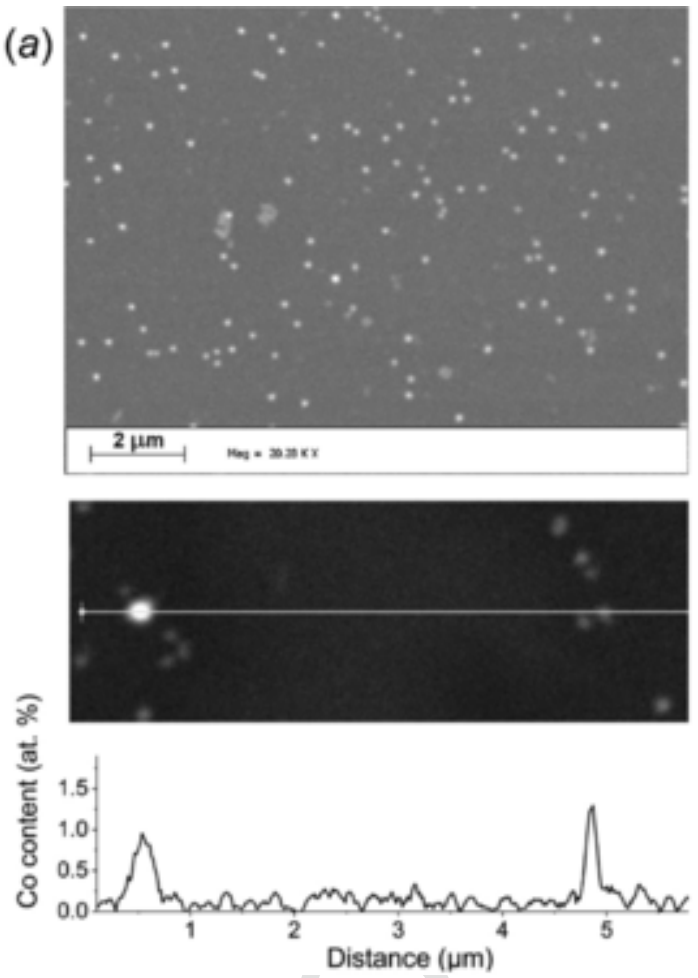

duced by the temperature dependent scattering by acoustic phonons in graphene [33,34].

The resistance of the pristine graphene sample appears to be 1780 $\mathrm{Ohm}$ /square at room temperature and $1960 \mathrm{Ohm}$ /square at $2 \mathrm{~K}$. The G/ CoNPs sample demonstrates a decreased $R_{\mathrm{sq}}$ value compared to the pristine graphene: $1040 \mathrm{Ohm} /$ square at room temperature and $1080 \mathrm{Ohm}$ / square at $2 \mathrm{~K}$. It correlates with the previously observed reduction of the graphene resistance after electrochemical deposition of Co NPs [19]. A decrease in $R_{\mathrm{sq}}$ has been explained by shunting the grain boundary barriers in polycrystalline graphene with Co particles, on the one hand, and by increasing the phase coherence length of carriers at the interface between graphene and Co particles, on the other hand [19].

The smallest $R_{\mathrm{sq}}$ values are observed for the G/CoNPs/G sample: $820 \mathrm{Ohm} /$ square at room temperature and $900 \mathrm{Ohm} /$ square at $2 \mathrm{~K}$. This cannot be explained by sole influence of Co particles, similarly to the G/CoNPs sample. Indeed, in the simple case of formation of bi-layer graphene with a pronounced electrical contact between the layers, the resistance should be decreased by half, as compared to each single

(b)
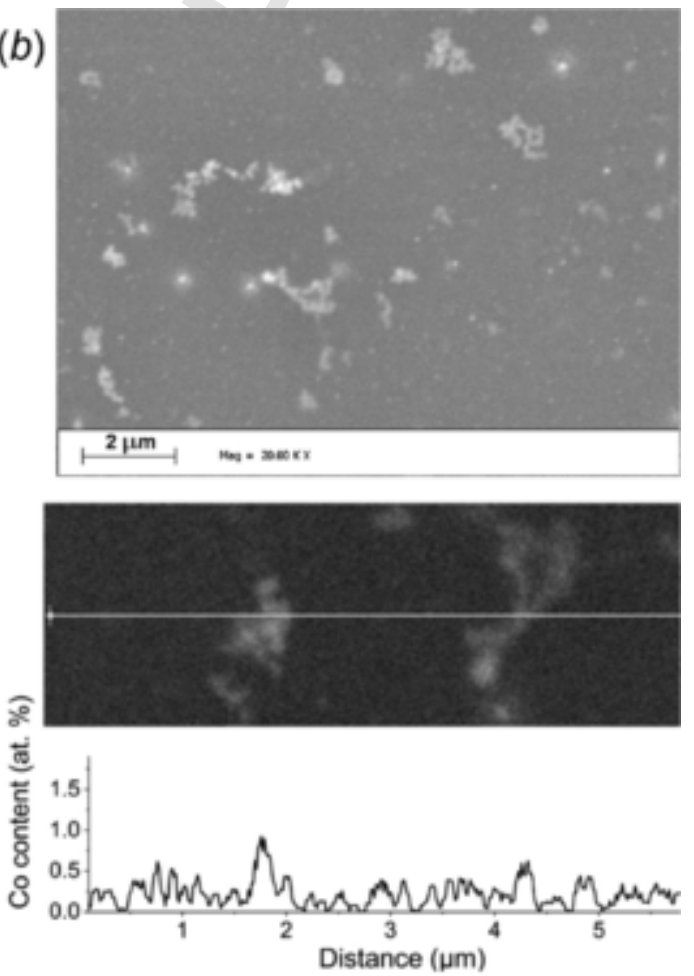

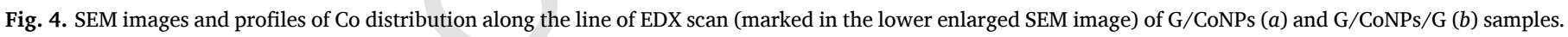
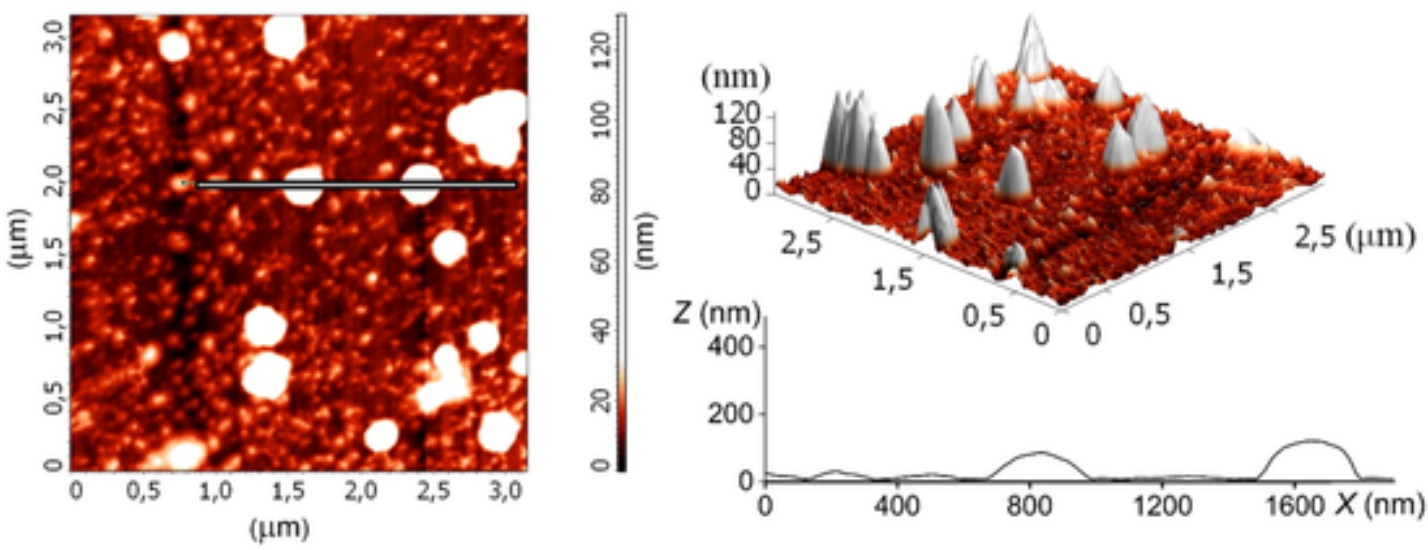

Fig. 5. 2D (left) and 3D (right) AFM images of G/CoNPs sample and the cross-section of surface morphology along the line marked on the 2D image. 

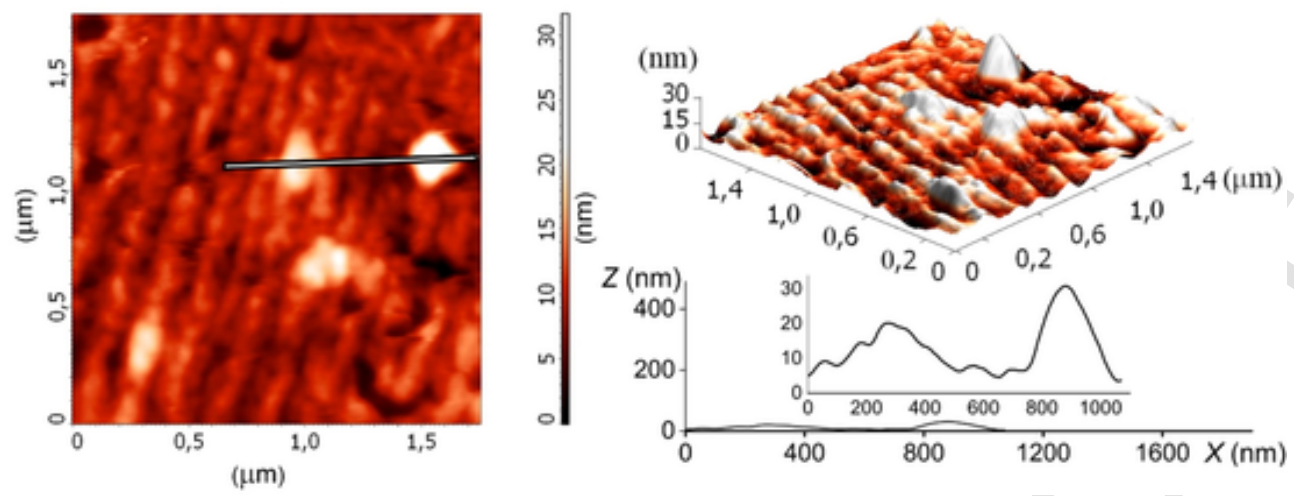

Fig. 6. 2D (left) and 3D (right) AFM images of G/CoNPs/G sample and the cross-section of surface morphology (lower panel) along the line marked on the 2D image.

(a)

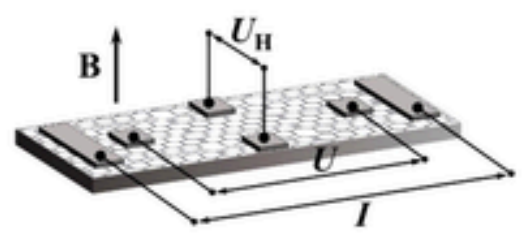

(b)
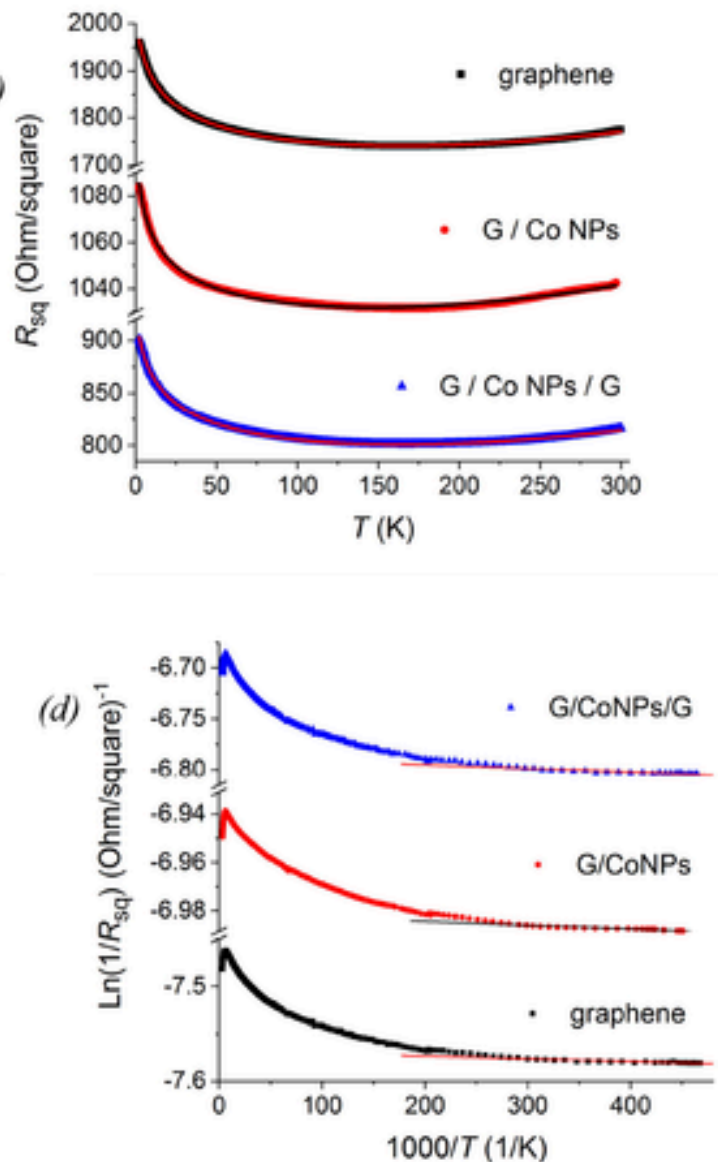

Fig. 7. Scheme of electrical contacts arrangement ( $a$ ) and the plots of $R_{\mathrm{sq}}$ vs. $T(b), \operatorname{Ln}\left(1 / R_{\mathrm{sq}}\right)$ vs. $T^{-1 / 3}(c)$ and $\operatorname{Ln}\left(1 / R_{\mathrm{sq}}\right)$ vs $(1000 / T)(d)$ dependences of graphene, $\mathrm{G} /$ CoNPs and G/CoNPs/G samples. The solid lines in plot $(b)$ are the best fits of the combination of expressions (1) and (2) and straight solid lines in plots $(c)$ and $(d)$ are the best fits to the Mott VRH $\left(1 / R^{\mathrm{VRH}}(T)\right)$ and the thermal activation $\left(1 / R^{\mathrm{TA}}(T)\right)$ models, respectively.

layer. However, as it was shown previously, Co NPs formed by electrochemical deposition are covered with the electrically insulating Co oxide shells [18] and, in consequence, cannot provide a good electrical contact between the bottom and top graphene layers, i.e. their parallel connection. In addition, taking into account the AFM results, which revealed a possible suspended state of the top graphene layer, such a contact is not also expected for other graphene regions, and the observed decrease in $R_{\mathrm{sq}}$ should not be the result of simple stacking of the graphene layers. On the other hand, the presence of regions with suspended graphene in the top graphene layer of the G/CoNPs/G sample could eliminate the substrate effect on carrier transport of this layer (scattering by charged imputities and surface phonons of substrate), thus redusing its resistivity. Looking ahead, this guess will be supported below by the results of the Hall effect and magnetoresistance measurements.

As it was mentioned above, all the samples demonstrate the presence of both the activation (semiconducting) and quasi-metallic types of electrical conductivity. The activation type (AT) of conductivity (i.e. the reciprocal of the resistivity) in graphene can be approximated by the sum of the Mott variable-range hopping (VRH) and thermal activation (TA) models $[35,36]$ : 


$$
\begin{aligned}
1 / R_{\mathrm{sq}}^{\mathrm{AT}}(T)= & 1 / R^{\mathrm{VRH}}(T)+1 / R^{\mathrm{TA}}(T) \\
= & \frac{1}{R_{\mathrm{VRH}}} \exp \left[-\left(\frac{T_{0}}{T}\right)^{1 / 3}\right] \\
& +\frac{1}{R_{\mathrm{TA}}} \exp \left[-\frac{E_{\mathrm{g}}}{2 k_{\mathrm{B}} T}\right]
\end{aligned}
$$

where $R_{\mathrm{VRH}}$ and $R_{\mathrm{TA}}$ are proportionality coefficients, $T$ - temperature, $k_{\mathrm{B}}-$ Boltzmann constant, $E_{\mathrm{g}}$ - the transport energy gap and $T_{0}=13.8 / k_{\mathrm{B}} N\left(E_{F}\right) L_{D}^{2}$ is the characteristic temperature related to the density of localized states at the Fermi energy $N\left(E_{\mathrm{F}}\right)$ and the electron localization length $L_{\mathrm{D}}$ [36].

From the other side, we have approximated the quasi-metallic (QM) behavior of electrical conductivity, visible at elevated temperatures, by the expression [34]:

$R_{\mathrm{sq}}^{\mathrm{QM}}(T)=R_{0}+\alpha T+\beta T^{2}$

This expression allows to approximate well the experimental $R_{\mathrm{sq}}(T)$ dependence in the temperature range from 150 to $300 \mathrm{~K}$. The increasing contribution of the quasi-metallic polynomial type resistance at high temperatures is generally associated with electron-phonon coupling effects inside the graphene grains, while the term of Mott VRH type resistance corresponds to the grain boundaries and defect states playing the role of barriers between the grains at lower temperatures (dominates from about $125 \mathrm{~K}$ down to $15 \mathrm{~K}$ ). The plots of the Mott VRH $\ln \left(1 / R_{\mathrm{sq}}\right)$ vs. $T^{-1 / 3}$ and TA $\ln \left(1 / R_{\mathrm{sq}}\right)$ vs. $1000 / T$ dependencies, shown in Fig. $7 \mathrm{c}$ and $7 \mathrm{~d}$, reveal the presence of the corresponding linear regions indicating that the experimental $R_{\mathrm{sq}}(T)$ can be represented as a superposition of different types of conductivity, including $1 / R^{\mathrm{VRH}}(T)$ and $1 / R^{\mathrm{TA}}$ (T) terms.

The results of approximation of $R_{\mathrm{sq}}(\mathrm{T})$ dependencies in the whole temperature range by the combination of expressions (1) and (2) are shown in Fig. $7 \mathrm{~b}$ as solid lines, while the fitted parameters are presented in Table 2. As one can note from Table 2, the values of the $\alpha$ parameter (corresponds to the linear term in (2)) is much smaller than $10^{-8} \mu \mathrm{Ohm} / \mathrm{K}$ and tends to zero. Thus, at elevated temperature, the quasi-metallic $R_{\mathrm{sq}}(\mathrm{T})$ behavior is predominantly represented by the $\beta T^{2}$ term that agrees well with the results described in $[37,38]$. The quadratic form of the temperature dependence is supposed to indicate weakened electron-phonon scattering in the studied samples of graphene, with electron-electron scattering being the dominant mechanism. The values of the characteristic temperature $T_{0}$ parameter in Mott VRH model are close for the pristine graphene $(77 \mathrm{~K}$ ) and G/CoNPs/G (70 K) samples, while for the $\mathrm{G} / \mathrm{CoNPs}$ sample the $T_{0}$ parameter appears to be two times smaller ( $31 \mathrm{~K}$ ).

Table 2

The parameters of $R_{\mathrm{sq}}(T)$ dependences approximation by the combination of expressions (1) and (2) for pristine graphene, modified graphene G/CoNPs and sandwich-like G/CoNPs/G samples: the characteristic temperature $T_{0}$, proportionality coefficients of Mott VRH $R_{\mathrm{VRH}}$ and thermal activation $R_{\mathrm{TA}}$ mechanisms, residual resistivity $R_{0}$, linear $(\alpha)$ and quadratic $(\beta)$ term coefficients of quasi-metallic mechanism.

\begin{tabular}{lllllll}
\hline Sample & $T_{0}, \mathrm{~K}$ & $\begin{array}{l}R_{\mathrm{VRH}}, \\
\text { Ohm/ } \\
\text { square }\end{array}$ & $\begin{array}{l}R_{\mathrm{TA}}, \mathrm{Ohm} / \\
\text { square }\end{array}$ & $\begin{array}{l}R_{0}, \mathrm{Ohm} / \\
\text { square }\end{array}$ & $\begin{array}{l}\alpha, 10^{-6} \\
\mathrm{Ohm} / \mathrm{K}\end{array}$ & $\begin{array}{l}\beta, 10^{-3} \\
\mathrm{Ohm} / \mathrm{K}^{2}\end{array}$ \\
\hline graphene & $77 \pm 4$ & $710 \pm 50$ & $810 \pm 30$ & $1180 \pm 30$ & $\ll 10^{-8}$ & $0.89 \pm 0.04$ \\
G/CoNPs & $31 \pm 5$ & $350 \pm 100$ & $260 \pm 30$ & $840 \pm 30$ & $\ll 10^{-8}$ & $0.24 \pm 0.02$ \\
G/ & $70 \pm 3$ & $420 \pm 30$ & $420 \pm 20$ & $509 \pm 15$ & $\ll 10^{-8}$ & $0.41 \pm 0.02$ \\
$\quad$ CoNPs/ & & & & & & \\
\multicolumn{1}{l}{ G } & & & & & & \\
\hline
\end{tabular}

\subsection{Hall effect}

The mobility and concentration of charge carriers were estimated by measuring the Hall effect in a magnetic field perpendicular to the surface of samples in the temperature range of $2-300 \mathrm{~K}$. Electric current of $10 \mu \mathrm{A}$ was applied using current-carrying contacts $(I)$, while the Hall voltage $U_{\mathrm{H}}$ was measured using voltage-sensing contacts $\left(U_{\mathrm{H}}\right)$ (Fig. $\left.7 \mathrm{a}\right)$.

The typical dependences of $U_{\mathrm{H}}$ on the applied magnetic field induction $B$ are presented in Fig. 8 a for the graphene, G/CoNPs and G/ CoNPs/G samples at $T=2 \mathrm{~K}$. The positive slopes of $U_{\mathrm{H}}(B)$ dependence with respect to $B$ axis indicate a predominantly positive charge of the carries in all the samples (p-type conductivity). The low-field Hall constants $R_{\mathrm{H}}$ are calculated using a linear approximation of the $U_{\mathrm{H}}(B)$ dependences according to the expression $U_{\mathrm{H}}=\left.R_{\mathrm{H}} \cdot \cdot B\right|_{I=10 \mu \mathrm{A}}$. The corresponding $R_{\mathrm{H}}(T)$ dependences are shown in Fig. 8b. As one can see from Fig. $8 \mathrm{~b}$, the $R_{\mathrm{H}}$ parameter of the G/CoNPs sample is reduced as compared to the pristine graphene sample in the whole temperature range studied $(2-300 \mathrm{~K})$. The latter should indicate an increase in the concentration $n$ of charge carriers, since $R_{\mathrm{H}}=1 /(e \cdot n)$, assuming that there is only one type of carrier (holes). However, the metal Co NPs cannot enrich the graphene with holes. An alternative possibility of the $R_{\mathrm{H}}$ decrease consists in doping of graphene with the carriers of the opposite sign (electrons), with the Co NPs being able to serve as a source of additional electrons. Therefore, two types of charge carriers should exist in the G/CoNPs sample.

Generally, in experiments on the Hall effect without an additional gate electrode, a polycrystalline CVD graphene should be considered as an ambipolar conductor with two types of charge carriers: electrons and holes [39-41]. As it was shown earlier, the grain boundaries tend to be doped mainly with n-type charge carriers, while the graphene grains are usually p-type doped [40,42]. For an ambipolar conductor, the Hall constant $R_{\mathrm{H}}$ can be expressed as

$R_{\mathrm{H}}=\frac{n_{\mathrm{h}} \mu_{\mathrm{h}}^{2}-\mathrm{n}_{\mathrm{e}} \mu_{\mathrm{e}}^{2}}{e\left(n_{\mathrm{h}} \mu_{\mathrm{h}}+\mathrm{n}_{\mathrm{e}} \mu_{\mathrm{e}}\right)^{2}}$

where $n_{\mathrm{h}}$ - surface charge density of the holes, $\mu_{\mathrm{h}}$ - hole mobility, $n_{\mathrm{e}}$ - surface charge density of the electrons, $\mu_{\mathrm{e}}$ - electron mobility, $e$ - elementary charge $\left(1.6 \times 10^{-19} \mathrm{C}\right)$.

Assuming the expression (3) for $R_{\mathrm{H}}$ of the ambipolar conductor, the $R_{\mathrm{H}}$ decrease could be explained by an increase in the surface charge density of electrons caused by ohmic contact between the graphene and Co NPs. At the same time, the $R_{\mathrm{H}}$ parameter increases significantly after covering the G/CoNPs sample with the top graphene layer (G/CoNPs/G sample), with the $R_{\mathrm{H}}$ values of the sandwich structure being higher than for the pristine graphene. Such an increase denotes either a change in the carrier mobility or a decrease in the surface charge density of electrons. As it was revealed earlier, Co NPs in the G/CoNPs sample have $\mathrm{CoO}$ shells [18]. So we do not expect the existence of ohmic contact between the top graphene layer and Co NPs in the G/CoNPs/G sample that could change the surface charge density of the electrons. Therefore, we suppose that the observed increase in the $R_{\mathrm{H}}$ parameter of the $\mathrm{G} / \mathrm{CoNPs} / \mathrm{G}$ sample is predominantly caused by an increase in the mobility of holes, see expression (3).

Since we cannot distinguish between the contributions of electrons and holes in the Hall experiment, the values of the surface charge density and charge mobility were estimated in terms of the effective $n^{*}$ and $\mu^{*}$ values, assuming that there is only one type of carrier (holes). For determining the effective surface charge density $n^{*}\left(\mathrm{~cm}^{-2}\right)$, we used the expression $R_{\mathrm{H}}=1 /\left(e \cdot n^{*}\right)$. The corresponding $n^{*}(T)$ dependences are presented in Fig. 8c. The values of the effective charge mobility $\mu^{*}$ at a given temperature were estimated using the corresponding values of sheet resistance $R_{\mathrm{sq}}$ (Fig. 7b) in the expression $\mu *=\mathrm{R}_{\mathrm{H}} / \mathrm{R}_{\mathrm{sq}}$ [43]. The $\mu^{*}$ ( $T$ ) dependences of the pristine and modified graphene samples are presented in Fig. 8d. It should be mentioned, that the $n^{*}$ parameter can be 
(a)

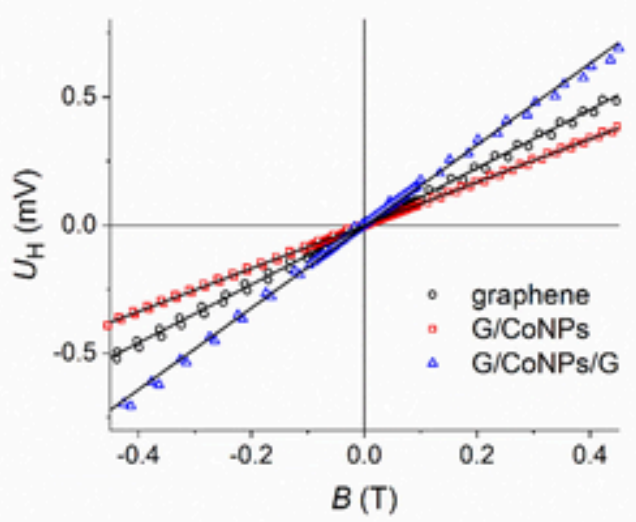

(c)

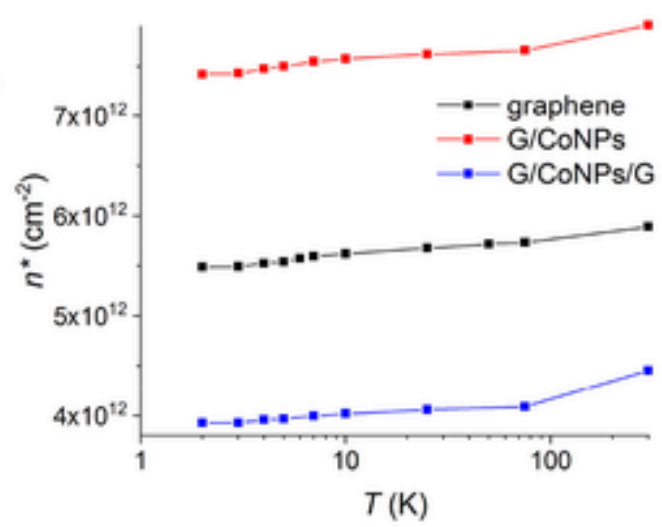

(b)

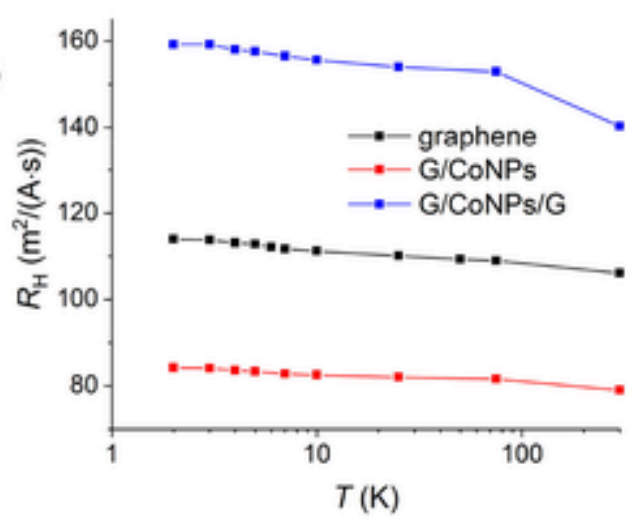

(d)

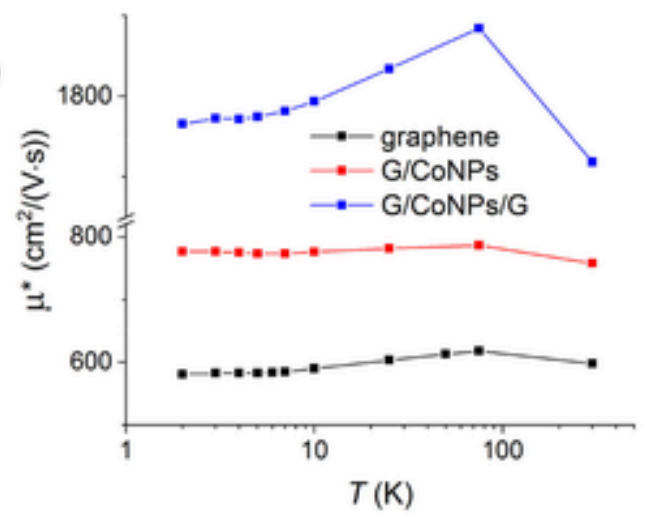

Fig. 8. The results of Hall effect measurements for graphene, G/CoNPs and G/CoNPs/G samples: $(a)$ Hall voltage $U_{\mathrm{H}}(B)$ dependences at 2 K, (b) Hall constants $R_{\mathrm{H}}$ (T) dependences, $(c)$ effective surface charge density $n^{*}(T)$ and $(d)$ effective charge mobility $\mu^{*}(T)$ dependences.

estimated adequately only for the pristine graphene and G/CoNPs/G samples, for which the assumption of one type carriers is applicable, since the contribution of electrons to their conductivity can be neglected. For the G/CoNPs sample, where the role of electrons to the carrier transport is essential, $n^{*}$ parameter shows nonphysical values. At the same time, the $\mu^{*}$ parameter as an effective characteristic of charge transport can be reasonably estimated for all the samples studied.

At the temperature $2 \mathrm{~K}$, the effective charge mobility in the pristine graphene is only $580 \mathrm{~cm}^{2} /(\mathrm{Vs})$. After electrochemical deposition of Co NPs it rises up to $780 \mathrm{~cm}^{2} /(\mathrm{Vs})$. An increase in $\mu^{*}$ could be caused by the formation of graphene regions with good ohmic contact with metal NPs that is supposed to decrease the scattering of charge carriers at the grain boundaries of polycrystalline CVD graphene located at these regions (shunting the grain boundaries). The covering of the G/CoNPs sample with the top graphene layer leads to the much bigger growth of $\mu^{*}$ up to $1800 \mathrm{~cm}^{2} /(\mathrm{V} \mathrm{s})$ at $2 \mathrm{~K}$. Such a significant increase in the carrier mobility was detected earlier for a turbostratic graphene in comparison with its single layer due to the eliminated influence of the substrate on the carrier transport $[21,22]$, with the $\mu$ values being close to the data obtained in this study. The currently revealed huge increase in $\mu^{*}$ confirms the aforementioned fact that the rise in the hole mobility is the main origin of the increased $R_{\mathrm{H}}$ value of the G/CoNPs/G sample.

The effective surface charge density $n^{*}$ in the graphene sample slightly increases with temperature from $5.5 \cdot 10^{12}$ at $2 \mathrm{~K}$ to $5.9 \cdot 10^{12}$ $\mathrm{cm}^{-2}$ at $300 \mathrm{~K}$. After deposition of Co NPs (G/CoNPs sample), it rises significantly up to $7.4 \cdot 10^{12} \mathrm{~cm}^{-2}$ at $2 \mathrm{~K}$. The growth of $n^{*}$ accompanied by the simultaneous increase in $\mu^{*}$ (Fig. 8d) does not correlate with the inverse relationship between the carrier mobility and surface charge density for CVD graphene with one type of carriers [44]. This result confirms the previous finding on the role of Co NPs in the charge transfer: shunting the grain boundary barriers in polycrystalline CVD graphene, on the one hand, and the change in phase coherence length of carriers at the interface between graphene and Co particles, on the other hand. Moreover, it serves as a source of additional charge carriers (electrons), thus violating the conditional assumption of one type of charge carrier in the studied samples.

The observed decrease in $n^{*}$ down to $3.9 \cdot 10^{12} \mathrm{~cm}^{-2}(T=2 \mathrm{~K})$ in the G/CoNPs/G sample, instead of its increase with respect to the pristine graphene, indicates indirectly that the electric current flows mainly in the top layer of the G/CoNPs/G structure, confirming the previous guess, that the decrease in the electrical resistance revealed could be explained by the presence of the areas of suspended graphene. Indeed, returning to the discussion of bi-layer graphene with a pronounced electrical contact between the layers, its surface charge density is supposed to be doubled, as compared to each layer on the assumption of their identity $[45,46]$. This is in contrast to the present observation. However, as it was shown earlier in $[21,22]$, adding the second monolayer of CVD graphene with a poor electrical contact with the bottom layer allows to obtain two-layered structure with negligible substrate effect on the transport of charge carriers in the top graphene layer. The quasifree state of the top layer, which does not contact either with the substrate or with the bottom layer, provides the improvement of its electrical transport properties, making them closer to ideal graphene that implies low surface charge density of carriers and their high mobility [26, 45]. Assuming the presence of regions with suspended graphene in our G/CoNPs/G sample, the observed decrease in $n^{*}$ with a simultaneous increase in $\mu^{*}$ could be due to the minimization of the substrate influence on the charge transport in graphene.

At low temperatures, an increase in $\mu^{*}$ is observed with increasing temperature. At room temperature, however, the effective charge mobility decreases in all the samples that is likely due to the temperaturedependent scattering of charge carriers (by each other or acoustic 
phonons). This effect agrees well with the quasi-metallic character of resistivity $R_{\mathrm{sq}}$ of the samples revealed earlier at room temperature (Fig. 7b) $[37,38]$.

We admit that the impact of Co NPs on charge transfer requires a deeper analysis of the Hall effect in the G/CoNPs sample on the assumption of the presence of two types of charge carriers: electrons in Co NPs and holes in graphene. As far as this paper is focused on electrical transport properties of the G/CoNPs/G structure, which are weakly dependent on the charge transfer in the bottom graphene layer, we limit the analysis of the Hall effect, assuming only one type of carriers.

\subsection{Magnetotransport properties}

The dependences of $R_{\mathrm{sq}}$ on the external magnetic field $B$ applied perpendicular to the surface of the samples were analyzed in terms of magnetoresistive (MR) effect determined as $\operatorname{MR}(B)=\left(R_{\mathrm{sq}}(B)-R_{\mathrm{sq}}(0)\right) / R_{\mathrm{sq}}(0)$, where $R_{\mathrm{sq}}(B)$ and $R_{\mathrm{sq}}(0)$ are the values of sheet resistance in non-zero and zero magnetic field, respectively. The $M R(B)$ dependences of the pristine and modified graphene samples measured at selected temperatures from 10 to $300 \mathrm{~K}$ are presented in Fig. 9. They are typical of the CVD graphene samples and demonstrate both positive (PMR) and negative (NMR) magnetoresistive effects [19].

The positive PMR effect, which dominates in strong magnetic field, is usually caused by Lorentz force acting on the charge carriers (socalled Lorentz mechanism). The PMR effect of the pristine graphene sample at $9 \mathrm{~T}$ reaches $103 \%$ at room temperature and gradually increases with decreasing temperature up to $123 \%$ at $10 \mathrm{~K}$. For the G/ CoNPs and G/CoNPs/G samples, the temperature dependences of PMR effect at $9 \mathrm{~T}$ are similar and consistent with the mechanism of Lorentz MR. The values of PMR effect of the G/CoNPs and G/CoNPs/G samples at $9 \mathrm{~T}$ at room temperature are $80 \%$ and $180 \%$, respectively. The more than 1.5 -fold increase in the high-field PMR value of the G/CoNPs/G

a)

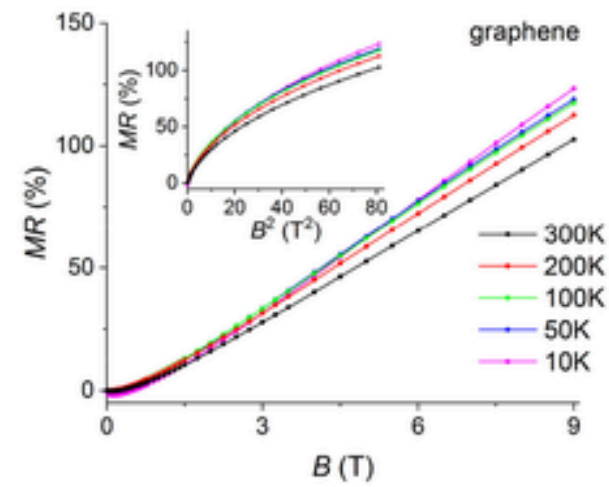

(c)

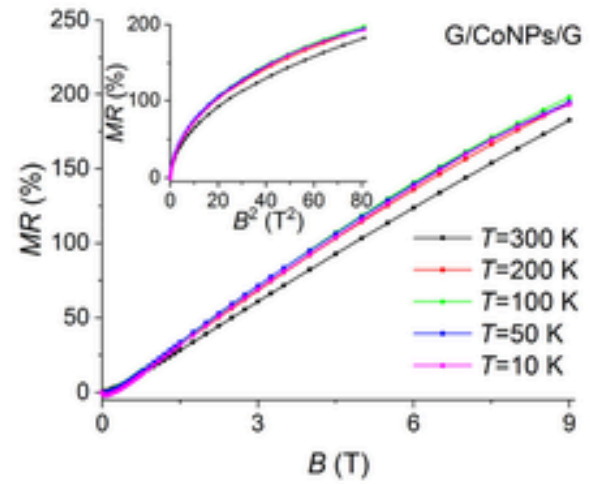

sample as compared to the pristine graphene is attributed to the suppressed effect of scattering due to the substrate on the carrier propagation path. The insets in Fig. 9 showing nonlinear $M R\left(B^{2}\right)$ dependences indicate the deviation of the $M R$ mechanism from the standard Lorentz model for one type of charge carriers described with $B^{2}$ field law. The latter assumes a more complex origin of the PMR effect typically associated with strong inhomogeneity in the carrier density [47].

The small NMR effect is observed at low magnetic fields (less than $0.5 \mathrm{~T}$ ) and low temperatures (less than $50 \mathrm{~K}$ ). The NMR effect in graphene is usually related to the field-induced suppression of quantum effects of coherent backscattering of charge carriers leading to their weak localization (WL), while the positive low-field MR contribution is well described by the effect of week antilocalization (WAL) $[48,49]$. To reveal the influence of graphene modifications on its transport properties determined by the mechanisms of charge carrier scattering, the low-field $M R(B)$ dependences were thoroughly analyzed for all the samples at $T=2-75 \mathrm{~K}$ (Fig. 10).

The $M R(B)$ dependences in Fig. 10 were analyzed on the assumption of quantum interference of charge carriers in monolayer graphene within the framework of the WL and WAL models described in $[48,50]$, using the following expression for $R_{\mathrm{sq}}(B)$ [51,52]:

(b)

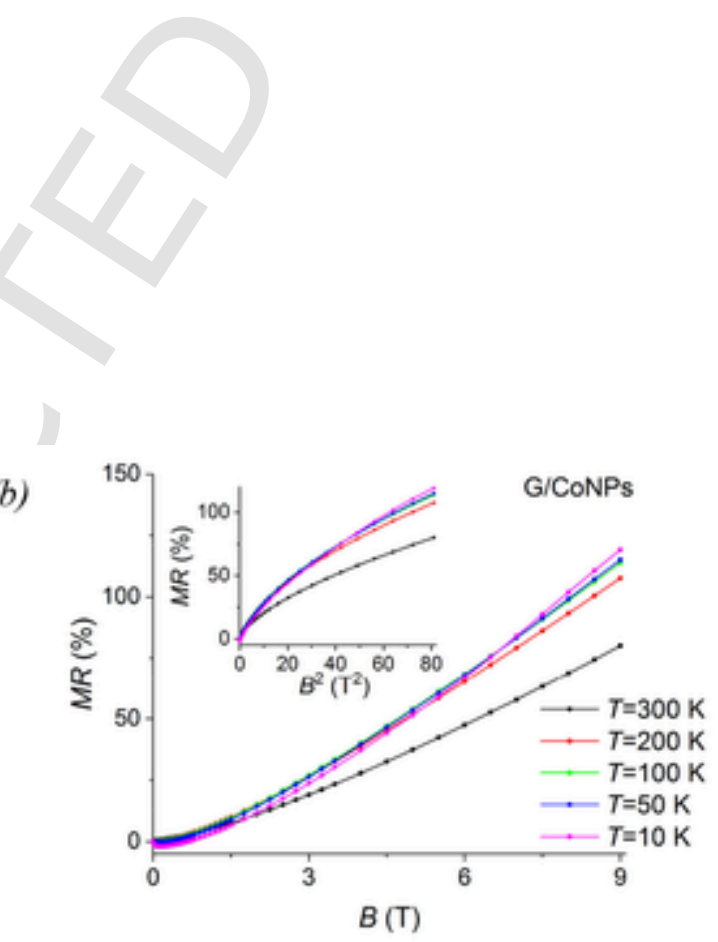


(a)

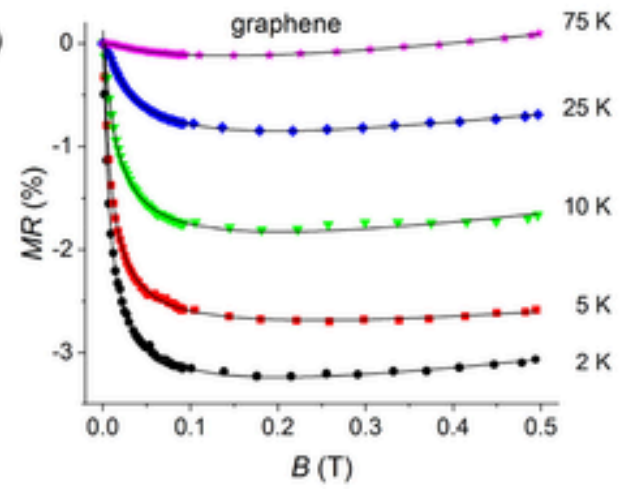

(c)

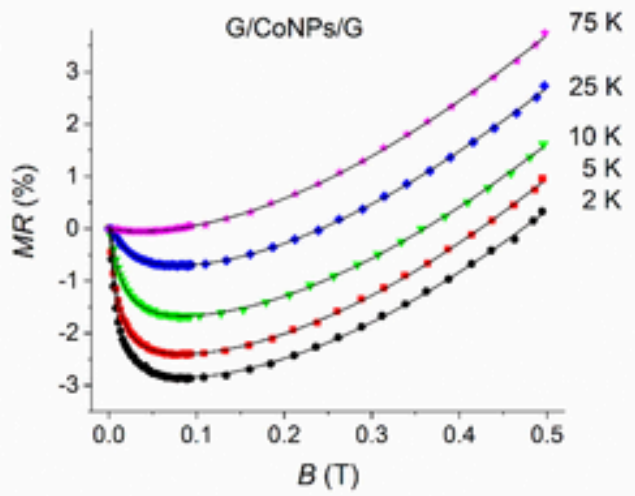

(b)

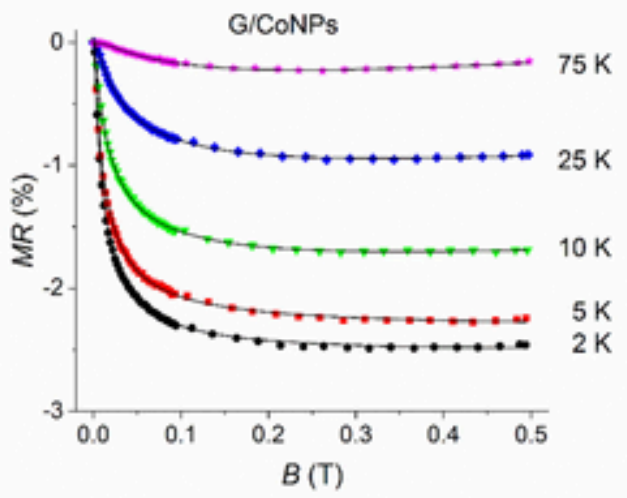

Fig. 10. The low-field $M R(B)$ dependences of ( $a$ ) graphene, $(b) \mathrm{G} /$ CoNPs and $(c) \mathrm{G} / \mathrm{CoNPs} / \mathrm{G}$ samples measured at low temperatures $(2-75 \mathrm{~K})$. The solid lines in plots (a)-(c) are the best fits of the expression (3).

$$
\begin{aligned}
\frac{1}{R_{\mathrm{sq}}(B)}=\frac{1}{R_{\mathrm{sq}}(0)}+\frac{e^{2}}{\pi \mathrm{h}}\left[F\left(\frac{8 \pi B}{\Phi_{0} L_{\phi}^{-2}}\right)\right. \\
\\
\quad-F\left(\frac{8 \pi B}{\Phi_{0}\left\{L_{\phi}^{-2}+2 L_{i}^{-2}\right\}}\right) \\
\left.-2 F\left(\frac{8 \pi B}{\Phi_{0}\left\{L_{\phi}^{-2}+L_{i}^{-2}+L_{*}^{-2}\right\}}\right)\right]
\end{aligned}
$$

Here $F(z)=\ln (z)+\psi\left(0.5+\mathrm{z}^{-1}\right), \psi(x)$ is the digamma function, $\Phi_{0}=h / e$ is the quantum of magnetic flux, $L_{\varphi}$ and $L_{i}$ are the phase coherence and elastic intervalley scattering lengths, respectively, $L *$ is the elastic intravalley scattering length. The scattering lengths $L_{\varphi, i}$,* relate to the corresponding scattering times $\tau_{\varphi, i, *}$ as $L_{\phi, i, *}=\sqrt{D \cdot \tau_{\phi, i, *}}$, where $D$ is the diffusion constant. The best fits of the $M R(B)$ dependences with the function (4) are shown with solid lines in Fig. 10 and the parameters of the obtained characteristic scattering lengths $L_{\varphi, i, *}$ are collected in Table 3.

The estimated values of the elastic intravalley scattering length $L$ * (Fig. 11a) are very close for all three samples $(\sim 20 \mathrm{~nm})$ and show rather weak temperature dependence. This parameter refers to the defectiveness of the crystalline structure of graphene, which causes the corresponding scattering of carrier on defects, [53] and does not depend on adding the Co NPs or on sandwich-like structure of the samples. In contrast, the phase coherence length $L_{\varphi}$ parameters show clear disparity at lowest temperatures and then converge with increasing $T$. They obey well the $T^{-1 / 2}$ dependence (Fig. 11b) indicating electron-electron interactions as the dominant dephasing mechanism in this temperature range $[54,55]$. The obtained $L_{\varphi}$ parameter determines the propagation
Table 3

The parameters obtained from fitting $M R(B)$ dependences with the equation (4): the elastic intravalley scattering length $L_{*}$, the phase coherence length $L_{\varphi}$ and the elastic intervalley scattering length $L_{i}$ in graphene, G/CoNPs and G/ CoNPs/G samples at temperatures 2-75 K.

\begin{tabular}{lllll}
\hline Sample & $T, \mathrm{~K}$ & $L_{\varphi}, \mathrm{nm}$ & $L_{i}, \mathrm{~nm}$ & $L_{*}, \mathrm{~nm}$ \\
\hline graphene & 2 & $511 \pm 4$ & $401 \pm 5$ & $20.3 \pm 0.5$ \\
& 5 & $430 \pm 2$ & $396 \pm 3$ & $18.3 \pm 0.4$ \\
& 10 & $310 \pm 3$ & $367 \pm 4$ & $21.8 \pm 0.3$ \\
& 25 & $195 \pm 2$ & $359 \pm 3$ & $21.9 \pm 0.2$ \\
& 75 & $117 \pm 2$ & $503 \pm 10$ & $22.0 \pm 0.2$ \\
G / Co NPs & 2 & $642 \pm 18$ & $254 \pm 16$ & $13 \pm 3$ \\
& 5 & $557 \pm 17$ & $211 \pm 8$ & $10 \pm 2$ \\
& 10 & $443 \pm 9$ & $174 \pm 5$ & $22.0 \pm 0.8$ \\
& 25 & $269 \pm 7$ & $143 \pm 7$ & $26.2 \pm 1.2$ \\
G / Co NPs / G & 75 & $108 \pm 6$ & $105 \pm 4$ & $21 \pm 7$ \\
& 2 & $340 \pm 3$ & $1760 \pm 170$ & $18.6 \pm 0.9$ \\
& 5 & $315 \pm 2$ & $1660 \pm 120$ & $19.4 \pm 0.7$ \\
& 10 & $257 \pm 2$ & $1490 \pm 80$ & $20.0 \pm 0.6$ \\
& 25 & $189 \pm 2$ & $1420 \pm 70$ & $21.0 \pm 0.5$ \\
& 75 & $112 \pm 14$ & $1800 \pm 300$ & $23.4 \pm 1.6$ \\
\hline
\end{tabular}

distance of electrons in graphene before they lose phase coherence due to inelastic scattering. An increase in the phase coherence length of carriers, associated with the suppression of decoherence mechanism, was mentioned above as a possible reason for the decreased $R_{\mathrm{sq}}$ values of the G/CoNPs sample (Fig. 7b) that is now confirmed by the estimate of the $L_{\varphi}$ parameter (Fig. 11b). As shown in Fig. 11 b, the $L_{\varphi}$ value decreases with temperature increase and becomes similar $(\sim 100 \mathrm{~nm})$ for all the samples at $T=75 \mathrm{~K}$, and the $L_{\varphi}$ parameter at higher temperatures should correspond to the length of electron free path [56]. At higher temperatures, when the quantum corrections to the graphene conductivity are not relevant more, an electron-electron dephasing mechanism of inelastic interaction turns into electron-electron scattering, 
(a)

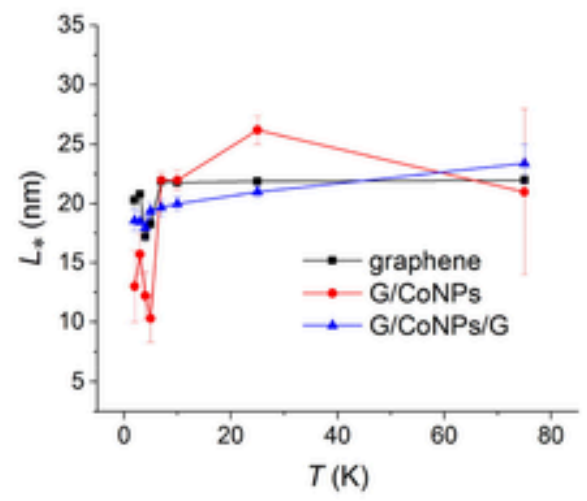

(c)

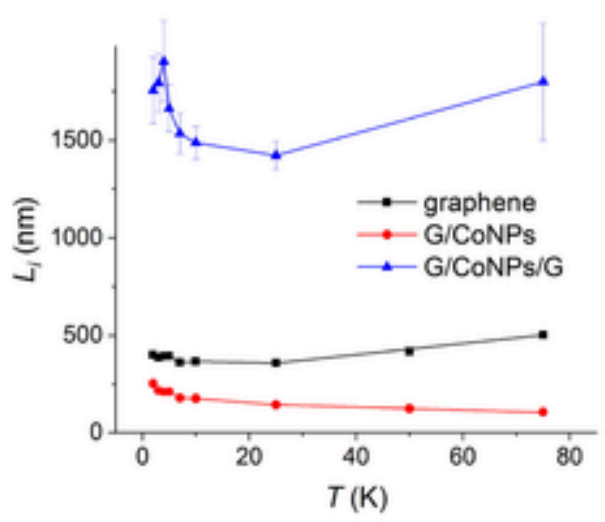

(b)

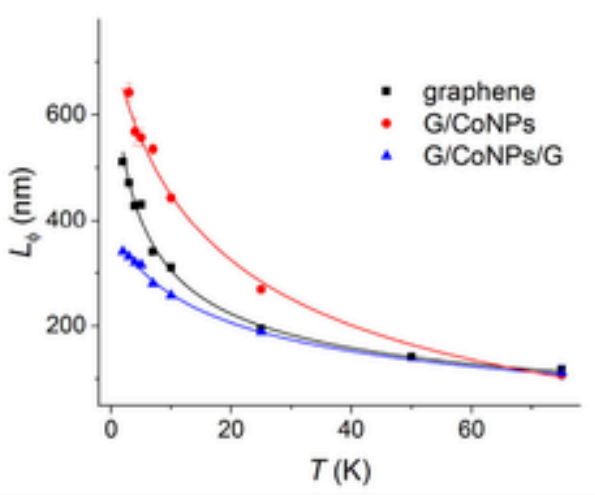

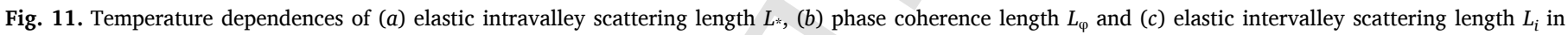

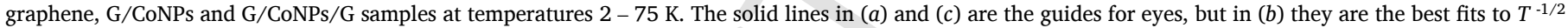
dependences.

which is confirmed by the approximation of the $R_{\mathrm{sq}}(T)$ dependences (Fig. 7b).

At the same time, a huge difference in the elastic intervalley scattering length $L_{i}$ is observed for the G/CoNPs/G sandwich in comparison with the graphene and G/CoNPs samples (Fig. 11c). In addition, the $L_{i}$ ( $T$ ) dependences for the pristine graphene and G/CoNPs/G sample show similar behavior, but different from that for the G/CoNPs sample. We suppose that much larger values of the elastic intervalley scattering length $L_{i}$ for the G/CoNPs/G sample are related to the presence of regions with suspended graphene in the top layer of the G/CoNPs/G sample, i.e. to the decreased interactions of its charge carriers with the scattering centers induced by the $\mathrm{SiO}_{2} / \mathrm{Si}$ substrate (e.g. charged impurities). Indeed, it is shown in $[48,51]$ that the increased $L_{i}$ length is inherent in graphene loosely attached to the substrate, with its $L_{\mathrm{i}}$ length being much higher than its $L_{\varphi}$ parameter [48]. Conversely, in the case of bi-layer graphene, these two lengths were found to be comparable [55]. It is important to mention, that the intervalley scattering process can be considered as a mobility-limiting factor [55]. This clarifies an evident correlation related to the proportional increase in the $L_{\mathrm{i}}$ and $\mu^{*}$ parameters of the G/CoNPs/G sample with respect to the pristine graphene. The value of the obtained $L_{\mathrm{i}}$ parameter of the G/CoNPs/G sample of the order of the graphene grain size indicates that scattering on the grain boundaries is the main factor limiting the carrier mobility in the sandwich-like structure studied.

\section{Summary and conclusions}

A new composite sandwich-like Graphene / Co nanoparticles / Graphene structure (G/CoNPs/G) was obtained on $\mathrm{SiO}_{2} / \mathrm{Si}$ or glass substrate by transferring a single layer of CVD graphene onto another single layer CVD graphene modified with Co nanoparticles (NPs). The Co NPs with mean diameter of $250 \mathrm{~nm}$ were deposited electrochemically.
According to the AFM results, the top graphene layer in this structure contains periodic ripples approximately $1 \mathrm{~nm}$ high. Non-contact suspended position of this layer above the bottom one is also indicated by a considerably lower height of the spacer Co NPs measured by AFM (through the top graphene layer). As it was estimated in our previous work, metallic cores of Co NPs are covered with insulating $\mathrm{CoO}$ oxide shells, which prevent the electrical contact between the NPs and top graphene layer.

According to the electrical transport measurements, the sheet resistance $R_{\text {sq }}$ of the G/CoNPs/G sample appears to be 2.2 times smaller than for the pristine graphene and 1.3 times smaller than for the G/CoNPs sample at room temperature. The dependences of $R_{\mathrm{sq}}$ on temperature of all the samples (graphene, G/CoNPs and G/CoNPs/G) demonstrate the presence of both the activation (semiconducting) and quasi-metallic contributions to the electrical conductivity, that can be well approximated by the combination of Mott variable-range hopping, thermal activation and quasi-metallic models. The fit of $R_{\mathrm{sq}}(T)$ dependences reveals that the quasi-metallic behavior of electrical conductivity is approximated as $R \sim T^{2}$. The characteristic temperature $T_{0}$ in the Mott variable-range hopping model related to the density of localized states at the Fermi energy and the electron localization length appears to be very close for the pristine graphene $(77 \mathrm{~K}$ ) and the G/CoNPs/G sample $(70 \mathrm{~K})$. At the same time, the parameter $T_{0}$ appears to be two times smaller $(31 \mathrm{~K})$ for the $\mathrm{G} /$ CoNPs sample that can be explained by the influence of Co particles. It means that the bottom graphene layer with Co NPs almost does not participate in the electrical conduction, which is dominated by the top graphene layer of the G/CoNPs/G sample.

Hall effect measurements revealed a p-type of conductivity as dominating in all the samples. Assuming two types of charge carriers, the decrease in the Hall constant $R_{\mathrm{H}}$ after deposition of Co NPs (G/CoNPs sample) indicates the increase in surface charge density of the electrons caused by Co NPs. The increase in $R_{\mathrm{H}}$ after covering the G/CoNPs sam- 
ple with the top graphene layer (G/CoNPs/G sample) is found to be due to the increase in the mobility of holes. In the approach of one type of charge carriers the effective charge mobility $\mu^{*}$ is estimated to be $1720 \mathrm{~cm}^{2} /(\mathrm{Vs})$ at room temperature for the G/CoNPs/G sample that is much larger than for the pristine graphene $\left(600 \mathrm{~cm}^{2} /(\mathrm{Vs})\right)$ as well as for the $\mathrm{G} / \mathrm{CoNPs}$ sample $\left(760 \mathrm{~cm}^{2} /(\mathrm{Vs})\right)$. At the same time, the corresponding effective surface charge density $n^{*}$ in the G/CoNPs/G sample is equal to $4.5 \times 10^{12} \mathrm{~cm}^{-2}$ at room temperature that is clearly smaller than for the pristine graphene $\left(5.9 \times 10^{12} \mathrm{~cm}^{-2}\right)$. Such changes in the $R_{\mathrm{H}}, \mu^{*}$ and $n^{*}$ parameters allow us to state that the revealed decrease in the electrical resistivity of the G/CoNPs/G sample can be explained by the presence of the areas of the suspended top graphene layer.

The analysis of magnetotransport properties of the graphene, G/ CoNPs and G/CoNPs/G samples revealed the presence of both positive (PMR) and negative (NMR) magnetoresistive effects. It is shown that the PMR effect observed in high magnetic fields ( $B$ greater than $0.5 \mathrm{~T}$ ) cannot be explained by a simple Lorentz mechanism for one type of charge carriers with square field $B^{2}$ dependence, indicating a more complex origin of the MR effect. The MR(B) dependences obtained at low temperatures $(2-75 \mathrm{~K}$ ) in low magnetic fields ( $B$ less than $0.5 \mathrm{~T}$ ) are successfully approximated on the assumption of quantum interference of charge carriers in graphene within the framework of the weak localization and antilocalization model. From the approximation, the parameters of elastic intravalley scattering length $L *$, phase coherence length $L_{\varphi}$ and elastic intervalley scattering length $L_{i}$ were estimated. The values of $L *$ length characterizing the defectiveness of graphene appear to be very similar and weakly temperature dependent for all three samples $(\sim 20 \mathrm{~nm})$. The phase coherence length $L_{\varphi}$ decreases with increasing temperature in agreement with $T^{-1 / 2}$ functional dependence indicating an electron-electron interaction as the dominating dephasing mechanism in all the samples studied at $T=2-75 \mathrm{~K}$. The most significant difference is observed for the parameter of $L_{i}$ length that appears to be much larger for the G/CoNPs/G sample than that for the pristine graphene and G/CoNPs samples. It is shown, that the revealed increase in the $L_{i}$ parameter relates to the reduced effect of the substrate on charge transport in the top graphene layer of the G/CoNPs/G structure due to its suspended state.

Assuming the above, the top graphene layer of the sandwich-like G/ CoNPs/G structure determines the electrical transport properties of the whole stack. The bottom layer does not participate in charge transport due to a suspended state of the top layer and the lack of electrical contact between the top layer and Co NPs provided by CoO shells. This approach of the sandwich-like G/CoNPs/G structure synthesis can be considered as an example of obtaining graphene based structures with eliminated substrate effect on charge transfer. The bottom graphene layer is used as an electrode for electrochemical deposition of NPs that further are used as a support for the top graphene layer. For developing flexible TCFs, useful in optoelectronic and magneto-optical applications, the bottom layer could previously be transferred on a transparent polymer substrate. The electrical transport properties in such sandwichlike structures will be predominantly determined by the quality of the top graphene layer that makes the proposed technology independent on the properties of the substrate used and promising for the mentioned application fields.

\section{CRediT authorship contribution statement}

V. Bayev: Supervision, Project administration, Conceptualization, Methodology, Formal analysis, Writing - original draft. M. Rybin: Investigation, Methodology, Validation. I. Svito: Investigation, Methodology, Validation. J. Przewoźnik: Investigation, Methodology, Validation. Cz. Kapusta: Writing review \& editing, Methodology. J. Kasiuk: Writing - review \& editing, Visualization. S. Vorobyova: Methodology, Re- sources. A. Konakov: Investigation, Methodology. E. Obraztsova: Methodology, Resources.

\section{Declaration of Competing Interest}

The authors declare that they have no known competing financial interests or personal relationships that could have appeared to influence the work reported in this paper.

\section{Acknowledgements}

The authors from Belarus acknowledge the financial support from the State Committee for Science and Technology of the Republic of Belarus (BRFFR project F18PLSHG-005). Maxim Rybin thanks Russian Federation President Program for young scientist MK-2370.2020.2. The authors of the paper are grateful to S.V. Gusakova and L.V Baran for their help in characterizing the samples by scanning electron microscopy and atomic force microscopy, as well as to Prof. A.K. Fedotov for critical comments and fruitful discussions.

\section{References}

[1] J.K. Wassei, R.B. Kaner, Graphene, a promising transparent conductor, Mater. Today 13 (3) (2010) 52-59.

[2] Resources Policy 52, 327 (2017)

[3] Z. Ding, Y. Zhu, C. Branford-White, K. Sun, S. Um-i-Zahra, J. Quan, H. Nie, L. Zhu, Self-assembled transparent conductive composite films of carboxylated multiwalled carbon nanotubes/poly(vinyl alcohol) electrospun nanofiber mats, Materials Lett. 128 (2014) 310-313, https://doi.org/10.1016/ j.matlet.2014.04.165.

[4] D. Zhang, K. Ryu, X. Liu, E. Polikarpov, J. Ly, M.E. Tompson, C. Zhou, Transparent, Conductive, and Flexible Carbon Nanotube Films and Their Application in Organic Light-Emitting Diodes, Nano Lett. 6 (9) (2006) 1880-1886, https://doi.org/10.1021/nl0608543.

[5] C. Chen, C. Ye, Metal Nanowires, in: D. Levy, E. Castellón (Eds.), Transparent Conductive Materials: Materials, Synthesis, Characterization, Applications, WileyVCH Verlag, Weinheim, 2018, pp. 105-131, https://doi.org/10.1002/ 9783527804603.ch2_3.

[6] J. Zou, H.-L. Yip, S.K. Hau, A.-Y. Jen, Metal grid/conducting polymer hybrid transparent electrode for inverted polymer solar cells, Appl. Phys. Lett. 96 (20) (2010) 203301, https://doi.org/10.1063/1.3394679.

[7] A. Patra, Y.H. Wijsboom, S.S. Zade, M. Li, Y. Sheynin, G. Leitus, M. Bendikov, Poly(3,4-ethylenedioxyselenophene), J. Am. Chem. Soc. 130 (21) (2008) 6734-6736, https://doi.org/10.1021/ja8018675.

[8] J. Tae Lim, H. Lee, H. Cho, B.-H. Kwon, N. Sung Cho, B. Kuk Lee, J. Park, J. Kim, J.-H. Han, J.-H. Yang, B.-G. Yu, C.-S. Hwang, S. Chu Lim, J.-I. Lee, Flexion bonding transfer of multilayered graphene as a top electrode in transparent organic lightemitting diodes, Scientific Rep. 5 (2015) 17748, https://doi.org/10.1038/ srep 17748.

[9] J.-W. Shin, J.-H. Han, H. Cho, J. Moon, B.-H. Kwon, S. Cho, T. Yoon, T.-S. Kim, M. Suemitsu, J.-I. Lee, N.S. Cho, Display process compatible accurate graphene patterning for OLED applications, 2D Mater. 5 (1) (2017) 014003, https://doi.org/ 10.1088/2053-1583/aa9cae.

[10] O.E. Kwon, J.-W. Shin, H. Oh, C.-m. Kang, H. Cho, B.-H. Kwon, C.-W. Byun, J.-H. Yang, K. Me Lee, J.-H. Han, N. Sung Cho, J. Hyuk Yoon, S. Jin Chae, J. Sung Park, H. Lee, C.-S. Hwang, J. Moon, J.-I. Lee, A prototype active-matrix OLED using graphene anode for flexible display application, J Information Display 21 (1) (2020) 49-56, https://doi.org/10.1080/15980316.2019.1680452.

[11] J. Moon, J.-W. Shin, H. Cho, J.-H. Han, N.S. Cho, J.T. Lim, S.K. Park, H.K. Choi, S.-Y. Choi, J.-H. Kim, M.-J. Maeng, J. Seo, Y. Park, J.-I. Lee, Technical issues in graphene anode organic light emitting diodes, Diamond and Related Mater. 57 (2015) 68-73, https://doi.org/10.1016/j.diamond.2015.03.020.

[12] Y. Xu, H. Yu, C. Wang, J. Cao, Y. Chen, Z. Ma, Y. You, J. Wan, X. Fang, X. Chen, Multilayer Graphene with Chemical Modification as Transparent Conducting Electrodes in Organic Light-Emitting Diode, Nanoscale Res. Lett. 12 (2017) 254, https://doi.org/10.1186/s11671-017-2009-9.

[13] D. Kim, D. Lee, Y. Lee, D.Y. Jeon, Work-Function Engineering of Graphene Anode by Bis(trifluoromethanesulfonyl)amide Doping for Efficient Polymer LightEmitting Diodes, Adv. Functional Mater. 23 (40) (2013) 5049-5055, https:// doi.org/10.1002/adfm201301386.

[14] K.K. Kim, A. Reina, Y. Shi, H. Park, L.-J. Li, Y.H. Lee, J. Kong, Enhancing the conductivity of transparent graphene films via doping, Nanotechnology 21 (28) (2010) 285205, https://doi.org/10.1088/0957-4484/21/28/285205.

[15] Y.i. Song, W. Fang, A.L. Hsu, J. Kong, Iron (III) Chloride doping of CVD graphene, Nanotechnology 25 (39) (2014) 395701, https://doi.org/10.1088/0957-4484/25/ 39/395701.

[16] M.G. Rybin, V.R. Islamova, E.A. Obraztsova, E.D. Obraztsova, Modification of graphene electronic properties via controllable gas-phase doping with copper chloride, Appl. Phys. Lett. 112 (3) (2018) 033107, https://doi.org/10.1063/ 
1.5006001

[17] R. Chen, S.R. Das, C. Jeong, M.R. Khan, D.B. Janes, M.A. Alam, Co-Percolating Graphene-Wrapped Silver Nanowire Network for High Performance, Highly Stable, Transparent Conducting Electrodes, Adv. Functional Mater. 23 (41) (2013) 5150-5158, https://doi.org/10.1002/adfm.201300124.

[18] V.G. Bayev, J.A. Fedotova, J.V. Kasiuk, S.A. Vorobyova, A.A. Sohor, I.V. Komissarov, N.G. Kovalchuk, S.L. Prischepa, N.I. Kargin, M. Andrulevičius, J. Przewoznik, C.z. Kapusta, O.A. Ivashkevich, S.I. Tyutyunnikov, N.N. Kolobylina, P.V. Guryeva, CVD graphene sheets electrochemically decorated with "core-shell" Co/CoO nanoparticles, Appl. Surf. Sci. 440 (2018) 1252-1260, https://doi.org/ 10.1016/j.apsusc.2018.01.245.

[19] A.K. Fedotov, S.L. Prischepa, J.A. Fedotova, V.G. Bayev, A.A. Ronassi, I.V. Komissarov, N.G. Kovalchuk, S.A. Vorobyova, O.A. Ivashkevich, Electrical conductivity and magnetoresistance in twisted graphene electrochemically decorated with Co particles, Phys. E: Low-dimensional Syst. Nanostruct. 117 (2020) 113790, https://doi.org/10.1016/j.physe.2019.113790.

[20] R.R. Nair, P. Blake, A.N. Grigorenko, K.S. Novoselov, T.J. Booth, T. Stauber, N.M.R. Peres, A.K. Geim, Fine Structure Constant Defines Visual Transparency of Graphene, Science 320 (5881) (2008) 1308, https://doi.org/10.1126/ science.1156965.

[21] K. Uemura, T. Ikuta, K. Maehashi, Turbostratic stacked CVD graphene for highperformance devices, Jpn. J. Appl. Phys. 57 (3) (2018) 030311, https://doi.org/ 10.7567/JJAP.57.030311.

[22] M. Shimatani, N. Yamada, S. Fukushima, S. Okuda, S. Ogawa, T. Ikuta, K. Maehashi, High-responsivity turbostratic stacked graphene photodetectors using enhanced photogating, Appl. Phys. Express 12 (12) (2019) 122010, https:// doi.org/10.7567/1882-0786/ab5096.

[23] M. Rybin, A. Pereyaslavtsev, T. Vasilieva, V. Myasnikov, I. Sokolov, A. Pavlova, E. Obraztsova, A. Khomich, V. Ralchenko, E. Obraztsova, Efficient nitrogen doping of graphene by plasma treatment, Carbon 96 (2016) 196-202, https://doi.org/ 10.1016/j.carbon.2015.09.056.

[24] A.R. Kim, R.G. Reddy, Cobalt Electrodeposition from Cobalt Chloride Using Urea and Choline Chloride Ionic Liquid: Effect of Temperature, Applied Voltage, and Cobalt Chloride Concentration on Current Efficiency and Energy Consumption, in: S. Wang, M. Free, S. Alam, M. Zhang, P. Taylor (Eds.), Applications of Process Engineering Principles in Materials Processing, Energy and Environmental Technologies, The Minerals, Metals \& Materials Series, Springer, Cham, 2017, pp. 97-114, https://doi.org/10.1007/978-3-319-51091-0 9.

[25] A. Das, S. Pisana, B. Chakraborty, S. Piscanec, S.K. Saha, U.V. Waghmare, K.S. Novoselov, H.R. Krishnamurthy, A.K. Geim, A.C. Ferrari, A.K. Sood, Monitoring dopants by Raman scattering in an electrochemically top-gated graphene transistor, Nature Nanotech. 3 (4) (2008) 210-215, https://doi.org/10.1038/ nnano.2008.67.

[26] K.S. Novoselov, Nobel Lecture: Graphene: Materials in the Flatland, Rev. Mod. Phys. 83 (3) (2011) 837-849, https://doi.org/10.1103/RevModPhys.83.837.

[27] K. Naito, N. Yoshinaga, E. Tsutsumi, Y. Akasaka, Transparent conducting film composed of graphene and silver nanowire stacked layers, Synthetic Metals 175 (2013) 42-46, https://doi.org/10.1016/j.synthmet.2013.04.025.

[28] M.J. Allen, V.C. Tung, R.B. Kaner, Honeycomb Carbon: A Review of Graphene, Chem. Reviews 110 (1) (2010) 132-145, https://doi.org/10.1021/cr900070d.

[29] P.R. Shaina, M. Jaiswal, Strain and morphology of graphene membranes on responsive microhydrogel patterns, J. Appl. Phys. 105 (19) (2014) 193103, https:// doi.org/10.1063/1.4901746.

[30] J.C. Meyer, A.K. Geim, M.I. Katsnelson, K.S. Novoselov, D. Obergfell, S. Roth, C. Girit, A. Zettl, On the roughness of single- and bi-layer graphene membranes, Solid State Commun. 143 (1-2) (2007) 101-109, https://doi.org/10.1016/ j.ssc.2007.02.047.

[31] J.C. Meyer, A.K. Geim, M.I. Katsnelson, K.S. Novoselov, T.J. Booth, S. Roth, The structure of suspended graphene sheets, Nature 446 (7131) (2007) 60-63, https:// doi.org/10.1038/nature05545.

[32] A. Isacsson, A.W. Cummings, L. Colombo, L. Colombo, J.M. Kinaret, S. Roche, Scaling properties of polycrystalline graphene: a review, 2D Mater. 4 (1) (2016) 012002, https://doi.org/10.1088/2053-1583/aa5147.

[33] J. Heo, H.J. Chung, S.-H. Lee, H. Yang, D.H. Seo, J.K. Shin, U.-I. Chung, S. Seo, E.H. Hwang, S. Das Sarma, Nonmonotonic temperature dependent transport in graphene grown by chemical vapor deposition, Phys. Rev. B 84 (2011) 035421 , https://doi.org/10.1103/PhysRevB.84.035421.

[34] M.H. Kang, G. Qiu, B. Chen, A. Jouvray, K.B.K. Teo, C. Cepek, L. Wu, J. Kim, W.I Milne, M.T. Cole, Transport in polymer-supported chemically-doped CVD graphene, J. Mater. Chem. C 5 (38) (2017) 9886-9897.

[35] H. Miyazaki, K. Tsukagoshi, A. Kanda, M. Otani, S. Okada, Influence of Disorder on Conductance in Bilayer Graphene under Perpendicular Electric Field, Nano Letters 10 (10) (2010) 3888-3892, https://doi.org/10.1021/nl1015365.
[36] A.L. Friedman, C.D. Cress, S.W. Schmucker, J.T. Robinson, O.M.J. van't Erve, Electronic transport and localization in nitrogen-doped graphene devices using hyperthermal ion implantation, Phys. Rev. B 93 (16) (2016), https://doi.org/ 10.1103/PhysRevB.93.161409.

[37] B. Davaji, H.D. Cho, M. Malakoutian, J.-K. Lee, G. Panin, T.W. Kang, C.H. Lee, A patterned single layer graphene resistance temperature sensor, Sci. Rep. 7 (2017) 8811, https://doi.org/10.1038/s41598-017-08967-y.

[38] E.H. Hwang, S. Das Sarma, Acoustic phonon scattering limited carrier mobility in two-dimensional extrinsic graphene, Phys. Rev. B 77 (2008) 115449, https:// doi.org/10.1103/PhysRevB.77.115449.

[39] A.H. Castro Neto, F. Guinea, N.M.R. Peres, K.S. Novoselov, A.K. Geim, The electronic properties of graphene, Rev. Mod. Phys. 81 (1) (2009) 109-162, https:// doi.org/10.1103/RevModPhys.81.109.

[40] A. Nachawaty, M. Yang, W. Desrat, S. Nanot, B. Jabakhanji, D. Kazazis, R. Yakimova, A. Cresti, W. Escoffier, B. Jouault, Magnetic field driven ambipolar quantum Hall effect in epitaxial graphene close to the charge neutrality point, Phys. Rev. B 96 (2017) 075442, https://doi.org/10.1103/PhysRevB.96.075442.

[41] M.F. Craciun, S. Russo, M. Yamamoto, S. Tarucha, Tuneable electronic properties in graphene, Nano Today 6 (1) (2011) 42-60, https://doi.org/10.1016/ j.nantod.2010.12.001.

[42] Oleg V. Yazyev, Yong P. Chen, Polycrystalline graphene and other twodimensional materials, Nature Nanotech. 9 (10) (2014) 755-767, https://doi.org/ 10.1038/nnano.2014.166.

[43] C.K. Safeer, Josep Ingla-Aynés, Franz Herling, José H. Garcia, Marc Vila, Nerea Ontoso, M.R. Calvo, Stephan Roche, Luis E. Hueso, Fèlix Casanova, RoomTemperature Spin Hall Effect in Graphene/ $\mathrm{MoS}_{2}$ van der Waals Heterostructures, Nano Lett. 19 (2) (2019) 1074-1082, https://doi.org/10.1021/ acs.nanolett.8b0436810.1021/acs.nanolett.8b04368.s001.

[44] N. Richter, Y.R. Hernandez, S. Schweitzer, J.-S. Kim, A.K. Patra, J. Englert, I. Lieberwirth, A. Liscio, V. Palermo, X. Feng, A. Hirsch, K. Müllen, M. Kläui, Robust Two-Dimensional Electronic Properties in Three-Dimensional Microstructures of Rotationally Stacked Turbostratic Graphene, Phys. Rev. Appl. 7 (2017) 024022, https://doi.org/10.1103/PhysRevApplied.7.024022.

[45] Kosuke Nagashio, Tomonori Nishimura, Koji Kita, Akira Toriumi, Mobility Variations in Mono- and Multi-Layer Graphene Films, Appl. Phys. Express 2 (2009) 025003, https://doi.org/10.1143/APEX.2.025003.

[46] W. Zhu, V. Perebeinos, M. Freitag, P. Avouris, Carrier scattering, mobilities, and electrostatic potential in monolayer, bilayer, and trilayer graphene, Phys. Rev. B 80 (2009) 235402, https://doi.org/10.1103/PhysRevB.80.235402.

[47] M.M. Parish, P.B. Littlewood, Non-saturating magnetoresistance in heavily disordered semiconductors, Nature 426 (6963) (2003) 162-165, https://doi.org/ 10.1038 /nature02073.

[48] E. McCann, K. Kechedzhi, Vladimir I. Fal'ko, H. Suzuura, T. Ando, B.L. Altshuler, Weak-Localization Magnetoresistance and Valley Symmetry in Graphene, Phys. Rev. Lett. 97 (14) (2006), https://doi.org/10.1103/PhysRevLett.97.146805.

[49] B.L. Altshuler, D. Khmel'nitzkii, A.I. Larkin, P.A. Lee, Magnetoresistance and Hall effect in a disordered two-dimensional electron gas, Phys. Rev. B 22 (11) (1980) 5142-5153, https://doi.org/10.1103/PhysRevB.22.5142.

[50] Vladimir I. Fal'ko, K. Kechedzhi, E. McCann, B.L. Altshuler, H. Suzuura, T. Ando, Weak localization in graphene, Solid State Commun. 143 (1-2) (2007) 33-38, https://doi.org/10.1016/j.ssc.2007.03.049.

[51] Nam-Hee Kim, Yun-Sok Shin, Serin Park, Hong-Seok Kim, Jun Sung Lee, Chi Won Ahn, Jeong-O Lee, Yong-Joo Doh, Quantum interference effects in chemical vapor deposited graphene, Current, Appl. Phys. 16 (1) (2016) 31-36, https://doi.org/ 10.1016/j.cap.2015.10.007.

[52] D.-K. Ki, D. Jeong, J.-H. Choi, H.-J. Lee, K.-S. Park, Inelastic scattering in a monolayer graphene sheet: A weak-localization study, Phys. Rev. B 78 (2008) 125409, https://doi.org/10.1103/PhysRevB.78.125409.

[53] F.V. Tikhonenko, D.W. Horsell, R.V. Gorbachev, A.K. Savchenko, Weak Localization in Graphene Flakes, Phys. Rev. Lett. 100 (2008) 056802, https:// doi.org/10.1103/PhysRevLett.100.056802.

[54] F.V. Tikhonenko, A.A. Kozikov, A.K. Savchenko, R.V. Gorbachev, Transition between Electron Localization and Antilocalization in Graphene, Phys. Rev. Lett. 103 (2009) 226801, https://doi.org/10.1103/PhysRevLett.103.226801.

[55] S. Engels, B. Terrés, A. Epping, T. Khodkov, K. Watanabe, T. Taniguchi, B. Beschoten, C. Stampfer, Limitations to Carrier Mobility and Phase-Coherent Transport in Bilayer Graphene, Phys. Rev. Lett. 113 (2014) 126801, https:// doi.org/10.1103/PhysRevLett.113.126801.

[56] H.-Z. Lu, W. Yao, D. Xiao, S.-Q. Shen, Intervalley Scattering and Localization Behaviors of Spin-Valley Coupled Dirac Fermions, Phys. Rev. Lett. 110 (2013) 016806, https://doi.org/10.1103/PhysRevLett.110.016806. 